\title{
ON THE SOLVABILITY \\ OF INITIAL-BOUNDARY VALUE PROBLEMS \\ FOR A VISCOUS COMPRESSIBLE FLUID IN AN INFINITE TIME INTERVAL
}

\author{
V. A. SOLONNIKOV \\ Dedicated to Nina Nicolaevna Ural'tseva \\ with great admiration
}

\begin{abstract}
The solution of the first boundary-value problem for the Navier-Stokes equations is estimated in the case of a compressible fluid in an infinite time interval; the solvability of the problem is proved, together with the exponential decay of the solution as $t \rightarrow \infty$. The proof is based on the "free work" method due to Prof. M. Padula. It is shown that the method is applicable to the analysis of free boundary problems.
\end{abstract}

\section{$\S 1$. INTRODUCTION}

This paper is devoted to the problem

$$
\left\{\begin{array}{l}
\rho(x, t)\left(\boldsymbol{v}_{t}+(\boldsymbol{v} \cdot \nabla) \boldsymbol{v}\right)-\nabla \cdot T(\boldsymbol{v})+\nabla p(\rho)=0, \\
\rho_{t}+\nabla \cdot(\rho \boldsymbol{v})=0, \quad x \in \Omega, \quad t>0, \\
\boldsymbol{v}(x, t)=0, \quad x \in S=\partial \Omega, \quad t>0, \\
\boldsymbol{v}(x, 0)=\boldsymbol{v}_{0}(x), \quad \rho(x, 0)=\rho_{0}(x), \quad x \in \Omega,
\end{array}\right.
$$

where $\Omega$ is a bounded domain in $\mathbb{R}^{3}, \boldsymbol{v}$ and $\rho$ are the velocity and the density of the fluid, $p(\rho)$ is a positive and strictly monotone increasing pressure function, $T(\boldsymbol{v})=\mu S(\boldsymbol{v})+$ $\mu_{1} I \nabla \cdot \boldsymbol{v}$ is a viscous part of the stress tensor, $S(\boldsymbol{v})=(\nabla \boldsymbol{v})+(\nabla v)^{T}$ is the doubled rate-of-strain tensor, and $\mu$ and $\mu_{1}$ are positive constant viscosity coefficients.

Problem (1.1) is very well studied (see [13, 14, 15, 16, 17, 18, 19]). In 1, 2, for arbitrarily large initial data M. Padula proved the inequality

$$
e^{2 \beta t}\left(\|\boldsymbol{v}(\cdot, t)\|_{L_{2}(\Omega)}^{2}+\|\rho(\cdot, t)-\bar{\rho}\|_{L_{2}(\Omega)}^{2}\right) \leq c\left(\left\|\boldsymbol{v}_{0}\right\|_{L_{2}(\Omega)}^{2}+\left\|\rho_{0}-\bar{\rho}_{0}\right\|_{L_{2}(\Omega)}^{2}\right), \quad t>0,
$$

where $\bar{\rho}=|\Omega|^{-1} \int_{\Omega} \rho(x, t) d x$ is the mean value of the density, $\beta, c=$ const $>0$. This inequality was obtained as a result of estimation of a certain auxiliary Lyapunov type function called "modified energy". It was assumed in [1, 2] that $p(\rho)=k \rho$. A similar estimate, in the case of a general $p(\rho)$, was obtained by Denisova in [3] for the problem of motion of two liquids of different types (compressible and incompressible) separated by a free interface and contained in a bounded vessel. Our goal is to obtain similar estimates for higher order derivatives of the solution. It has turned out that the same method is applicable for doing this. We also establish the solvability of the problem in an infinite

2010 Mathematics Subject Classification. Primary 35Q30.

Key words and phrases. Navier-Stokes equations, viscosity, anisotropic Sobolev-Slobodetski spaces.

The author is thankful to E. V. Frolova, W. M. Zajaczkowski, and V. Kalantarov for useful suggestions and discussions. The work was partially supported by the EU project FLUX 319012. 
time interval. But, in contrast to the result of M. Padula, we are constrained to require the smallness of the initial data.

We work in anisotropic Sobolev-Slobodetski spaces

$$
W_{2}^{r, r / 2}\left(\mathcal{D}_{T}\right)=W_{2}^{r, 0}\left(\mathcal{D}_{T}\right) \cap W_{2}^{0, r / 2}\left(\mathcal{D}_{T}\right),
$$

where $\mathcal{D}_{T}=\mathcal{D} \times(0, T), T \leq \infty, \mathcal{D}$ is a domain in $\mathbb{R}^{n}$,

$$
W_{2}^{r, 0}\left(\mathcal{D}_{T}\right)=L_{2}\left(0, T ; W_{2}^{r}(\mathcal{D})\right), \quad W_{2}^{0, s}\left(\mathcal{D}_{T}\right)=W_{2}^{s}\left(0, T ; L_{2}(\mathcal{D})\right) .
$$

By $W_{2}^{l}(\mathcal{D})$ we mean an isotropic Sobolev space with the norm given by

$$
\|u\|_{W_{2}^{l}(\mathcal{D})}^{2}=\sum_{|j| \leq l}\left\|D^{j} u\right\|_{L_{2}(\mathcal{D})}^{2}
$$

if $l$ is an integer, or

if $l=[l]+\lambda, 0<\lambda<1$.

$$
\|u\|_{W_{2}^{l}(\mathcal{D})}^{2}=\|u\|_{W_{2}^{[l]}(\mathcal{D})}^{2}+\sum_{|j|=[l]} \int_{\mathcal{D}} \int_{\mathcal{D}}\left|D^{j} u(x)-D^{j} u(y)\right|^{2} \frac{d x d y}{|x-y|^{n+2 \lambda}},
$$

We write problem (1.1) in the Lagrangian coordinates $\xi \in \Omega$ related to the Eulerian coordinates $x \in \Omega$ by

$$
x=\xi+\int_{0}^{t} \boldsymbol{u}(\xi, t) d \xi \equiv X(\xi, t)=\xi+\mathbf{U}(\xi, \mathbf{t}),
$$

where $\boldsymbol{u}(\xi, t)=\boldsymbol{v}(X(\xi, t), t)$. The transformation (1.3) converts the problem (1.1) into

$$
\left\{\begin{array}{l}
r \boldsymbol{u}_{t}-\nabla_{u} \cdot T_{u}(\boldsymbol{u})+\nabla_{u} p(r)=0, \\
r_{t}+r \nabla_{u} \cdot \boldsymbol{u}=0, \quad \xi \in \Omega, \quad \boldsymbol{u}(\xi, t)=0, \quad \xi \in S, \\
\boldsymbol{u}(\xi, 0)=\boldsymbol{v}_{0}(\xi), \quad r(\xi, 0)=\rho_{0}(\xi), \quad \xi \in \Omega,
\end{array}\right.
$$

where $r(\xi, t)=\rho(X(\xi, t), t), \nabla_{u}=\mathcal{L}^{-T} \nabla=J_{u}^{-1} A(\xi, t) \nabla$ is the transformed gradient $\nabla_{x}$, $\mathcal{L}=\left(\frac{\partial X}{\partial \xi}\right) . J_{u}=\operatorname{det} \mathcal{L}, A$ is the co-factors matrix of $\mathcal{L}$, and $T_{u}(\boldsymbol{u})=\mu S_{u}(\boldsymbol{u})+\mu_{1} I \nabla_{u} \cdot \boldsymbol{u}$, $S_{u}(\boldsymbol{u})=\left(\nabla_{u} \boldsymbol{u}\right)+\left(\nabla_{u} \boldsymbol{u}\right)^{T}$. The reason why the Lagrangian coordinates are used is purely technical: in these coordinates the space derivatives of $r$ disappear from the continuity equation.

We set $r=\bar{\rho}+\theta$, where $\bar{\rho}=M /|\Omega|$ is the mean density and $M=\int_{\Omega} \rho(x, t) d x=$ $\int_{\Omega} r(\xi, t) J_{u}(\xi, t) d \xi=$ const is the total mass of the fluid. Clearly, $\int_{\Omega} \theta(\xi, t) J_{u}(\xi, t) d \xi=0$. System (1.4) can be written in the form

$$
\left\{\begin{array}{l}
\bar{\rho} \boldsymbol{u}_{t}-\nabla \cdot T(\boldsymbol{u})+p^{\prime}(\bar{\rho}) \nabla \theta=\boldsymbol{l}_{1}(\boldsymbol{u}, \theta) \\
\theta_{t}+\bar{\rho} \nabla \cdot \boldsymbol{u}=l_{2}(\boldsymbol{u}, \theta), \quad \xi \in \Omega, \\
\boldsymbol{u}(\xi, t)=0, \quad \xi \in S, \\
\boldsymbol{u}(\xi, 0)=\boldsymbol{v}_{0}(\xi), \quad \theta(\xi, 0)=\theta_{0}(\xi)=\rho_{0}(\xi)-\bar{\rho},
\end{array}\right.
$$

where

$$
\begin{aligned}
& \boldsymbol{l}_{1}=-\theta \boldsymbol{u}_{t}+\left(\nabla_{u} \cdot T_{u}(\boldsymbol{u})-\nabla \cdot T(\boldsymbol{u})\right)+p^{\prime}(\bar{\rho}) \nabla \theta-p^{\prime}(\bar{\rho}+\theta) \nabla_{u} \theta, \\
& l_{2}=-r \nabla_{u} \cdot \boldsymbol{u}+\bar{\rho} \nabla \cdot \boldsymbol{u}=\bar{\rho}\left(\nabla-\nabla_{u}\right) \cdot \boldsymbol{u}-\theta \nabla_{u} \cdot \boldsymbol{u} .
\end{aligned}
$$

Replacing $\boldsymbol{l}_{1}$ and $l_{2}$ by some given functions $\boldsymbol{f}$ and $h$, we obtain a linear problem

$$
\left\{\begin{array}{l}
\bar{\rho} \boldsymbol{v}_{t}-\nabla \cdot T(\boldsymbol{v})+p^{\prime}(\bar{\rho}) \nabla \theta=\boldsymbol{f}(x, t), \\
\theta_{t}+\bar{\rho} \nabla \cdot \boldsymbol{v}=h(x, t), \quad x \in \Omega, \quad t>0, \\
\boldsymbol{v}(x, t)=0, \quad x \in S, \quad t>0, \\
\boldsymbol{v}(x, 0)=\boldsymbol{v}_{0}(x), \quad \theta(x, 0)=\theta_{0}(x), \quad x \in \Omega .
\end{array}\right.
$$

The main result of the paper is as follows. 
Theorem 1. Suppose $S \in W_{2}^{3 / 2+l}, 1 / 2<l<1, p \in C^{2}(\bar{\rho} / 2,3 \bar{\rho} / 2), \bar{\rho}, p^{\prime}(\bar{\rho})>0$, and $\left|\rho_{0}(x)\right| \geq c_{0}>0$. For arbitrary $\boldsymbol{v}_{0} \in W_{2}^{l+1}(\Omega)$ and $\theta_{0} \in W_{2}^{l+1}(\Omega)$ satisfying the conditions

$$
\begin{aligned}
& \boldsymbol{v}_{0}(x, 0)=0, \quad x \in S, \quad \int_{\Omega} \theta_{0}(x) d x=0, \\
& \left\|\boldsymbol{v}_{0}\right\|_{W_{2}^{l+1}(\Omega)}+\left\|\theta_{0}\right\|_{W_{2}^{l+1}(\Omega)} \leq \epsilon \ll 1
\end{aligned}
$$

problem (1.4) has a unique solution defined for $t \geq 0$, and

$$
\begin{array}{r}
\left\|e^{\beta t} \boldsymbol{u}\right\|_{W_{2}^{l+2, l / 2+1}\left(Q_{T}\right)}+\left\|e^{\beta t} \theta\right\|_{W_{2}^{l+1,0}\left(Q_{T}\right)}+\left\|e^{\beta t} \theta_{t}\right\|_{W_{2}^{l+1,0}\left(Q_{T}\right)} \\
\leq c\left(\left\|\boldsymbol{v}_{0}\right\|_{W_{2}^{l+1}(\Omega)}+\left\|\theta_{0}\right\|_{W_{2}^{l+1}(\Omega)}\right),
\end{array}
$$

where $Q_{T}=\Omega \times(0, T), T \leq \infty, \beta>0$.

The proof of this theorem is given in $\S 3 ; \S 2$ contains necessary estimates of the solution of a linearized problem. In $\S 4$, a somewhat more technical method of the analysis of the free boundary problem for two fluids is outlined. The results of $\S 2$ (in particular, estimate (2.2)) enable us to take account of the mass forces $\boldsymbol{f}(x, t)$ decaying exponentially as $t \rightarrow \infty$. We have not done it for the sake of simplicity.

\section{§2. Linear PROBLEM}

In this section we obtain the following result.

Theorem 2. Suppose that $\bar{\rho}, p^{\prime}(\bar{\rho})>0, \boldsymbol{v}_{0} \in W_{2}^{l+1}(\Omega), \theta_{0} \in W_{2}^{l+1}(\Omega), \boldsymbol{f} \in W_{2}^{l, l / 2}\left(Q_{T}\right)$, $h \in W_{2}^{l+1,0}\left(Q_{T}\right), T \leq \infty, 1 / 2<l<1$, and

$$
\int_{\Omega} h(x, t) d x=0, \quad \int_{\Omega} \theta_{0}(x) d x=0 .
$$

Then problem (1.7) has a unique solution

$$
\boldsymbol{v} \in W_{2}^{2+l, 1+l / 2}\left(Q_{T}\right), \quad \theta, \theta_{t} \in W_{2}^{l+1,0}\left(Q_{T}\right),
$$

and

$$
\begin{aligned}
& \left\|e^{\beta t} \boldsymbol{v}\right\|_{W_{2}^{l+2, l / 2+1}\left(Q_{T}\right)}+\left\|e^{\beta t} \theta\right\|_{W_{2}^{l+1,0}\left(Q_{T}\right)}+\left\|e^{\beta t} \theta_{t}\right\|_{W_{2}^{l+1,0}\left(Q_{T}\right)} \\
& \quad \leq c\left(\left\|\boldsymbol{v}_{0}\right\|_{W_{2}^{l+1}(\Omega)}+\left\|\theta_{0}\right\|_{W_{2}^{l+1}(\Omega)}+\left\|e^{\beta t} \boldsymbol{f}\right\|_{W_{2}^{l, l / 2}\left(Q_{T}\right)}+\left\|e^{\beta t} h\right\|_{W_{2}^{l+1,0}\left(Q_{T}\right)}\right)
\end{aligned}
$$

with $\beta>0$ and $c$ independent of $T \leq \infty$.

The proof of this theorem occupies the rest of the section; our main attention is given to the proof of (2.2). We start with reproducing an analog of M. Padula's result for the linear problem (1.7).

Proposition 1. If conditions (2.1) are fulfilled, then the solution of problem (1.7) satisfies the inequality

$$
\begin{aligned}
& \sup _{t<T} e^{2 \beta t}\left(\|\boldsymbol{v}(\cdot, t)\|_{L_{2}(\Omega)}^{2}+\|\theta(\cdot, t)\|_{L_{2}(\Omega)}^{2}\right)+\int_{0}^{T} e^{2 \beta t}\left(\|\boldsymbol{v}\|_{W_{2}^{1}(\Omega)}^{2}+\|\theta\|_{L_{2}(\Omega)}^{2}\right) d t \\
& \quad \leq c\left(\left\|\boldsymbol{v}_{0}\right\|_{L_{2}(\Omega)}^{2}+\left\|\theta_{0}\right\|_{L_{2}(\Omega)}^{2}+\int_{0}^{T} e^{2 \beta t}\left(\|\boldsymbol{f}\|_{L_{2}(\Omega)}^{2}+\|h\|_{L_{2}(\Omega)}^{2}\right) d t\right), \quad T \leq \infty .
\end{aligned}
$$

Proof. We note that conditions (2.1) imply $\int_{\Omega} \theta(x, t) d x=0, \quad t>0$. By standard calculation, the energy relation

$$
\frac{1}{2} \frac{d}{d t} \bar{\rho}\|\boldsymbol{v}\|_{L_{2}(\Omega)}^{2}+\int_{\Omega} T(\boldsymbol{v}): \nabla \boldsymbol{v} d x-p^{\prime}(\bar{\rho}) \int_{\Omega} \theta \nabla \cdot \boldsymbol{v} d x=\int_{\Omega} \boldsymbol{f} \cdot \boldsymbol{v} d x
$$


can be obtained, which implies

$$
\begin{array}{r}
\frac{1}{2} \frac{d}{d t}\left(\bar{\rho}\|\boldsymbol{v}\|_{L_{2}(\Omega)}^{2}+\frac{p^{\prime}(\bar{\rho})}{\bar{\rho}}\|\theta\|_{L_{2}(\Omega)}^{2}\right)+\frac{\mu}{2}\|S(\boldsymbol{v})\|_{L_{2}(\Omega)}^{2}+\mu_{1}\|\nabla \cdot \boldsymbol{v}\|_{L_{2}(\Omega)}^{2} \\
=\int_{\Omega}\left(\boldsymbol{f} \cdot \boldsymbol{v}+\frac{p^{\prime}(\bar{\rho})}{\bar{\rho}} h \theta\right) d x .
\end{array}
$$

Next, we introduce the vector field $\boldsymbol{V}(x, t)$ such that

$$
\begin{aligned}
& \nabla \cdot \boldsymbol{V}(x, t)=-\theta(x, t), \quad x \in \Omega, \quad \boldsymbol{V}(x, t)=0, \quad x \in S, \\
& \|\boldsymbol{V}\|_{W_{2}^{1}(\Omega)} \leq c\|\theta\|_{L_{2}(\Omega)}, \\
& \left\|\boldsymbol{V}_{t}\right\|_{W_{2}^{1}(\Omega)} \leq c\left\|\theta_{t}\right\|_{L_{2}(\Omega)} \leq c\left(\|\boldsymbol{v}\|_{W_{2}^{1}(\Omega)}+\|h\|_{L_{2}(\Omega)}\right)
\end{aligned}
$$

(problem (2.5) has been analyzed by many authors, see, e.g., [4, 5, 6]). We multiply the first equation in (1.7) by $\boldsymbol{V}$ and integrate over $\Omega$, obtaining

$$
\bar{\rho}\left(\frac{d}{d t} \int_{\Omega} \boldsymbol{v} \cdot \boldsymbol{V} d x-\int_{\Omega} \boldsymbol{v} \cdot \boldsymbol{V}_{t} d x\right)+\int_{\Omega} T(\boldsymbol{v}): \nabla \boldsymbol{V} d x+p^{\prime}(\bar{\rho})\|\theta\|_{L_{2}(\Omega)}^{2}=\int_{\Omega} \boldsymbol{f} \cdot \boldsymbol{V} d x
$$

Finally, we multiply (2.6) by a small positive $\gamma$ and add to (2.4), which yields

$$
\frac{1}{2} \frac{d E_{0}(t)}{d t}+E_{1}(t)=\int_{\Omega}\left(\boldsymbol{f} \cdot(\boldsymbol{v}+\gamma \boldsymbol{V})+\frac{p^{\prime}(\bar{\rho})}{\bar{\rho}} h \theta\right) d x+\bar{\rho} \gamma \int_{\Omega} \boldsymbol{v} \cdot \boldsymbol{V}_{t} d x
$$

where

$$
\begin{aligned}
& E_{0}(t)=\bar{\rho}\|\boldsymbol{v}\|_{L_{2}(\Omega)}^{2}+\frac{p^{\prime}(\bar{\rho})}{\bar{\rho}}\|\theta\|_{L_{2}(\Omega)}^{2}+2 \bar{\rho} \gamma \int_{\Omega} \boldsymbol{v} \cdot \boldsymbol{V} d x \\
& E_{1}(t)=\frac{\mu}{2}\|S(\boldsymbol{v})\|_{L_{2}(\Omega)}^{2}+\mu_{1}\|\nabla \cdot \boldsymbol{v}\|_{L_{2}(\Omega)}^{2}+\gamma p^{\prime}(\bar{\rho})\|\theta\|_{L_{2}(\Omega)}^{2}+\gamma \int_{\Omega} T(\boldsymbol{v}): \nabla \boldsymbol{V} d x .
\end{aligned}
$$

Due to $(2.5),(2.8)$ and the Korn inequality, we have

$$
\begin{aligned}
& c_{1}\left(\|\boldsymbol{v}\|_{L_{2}(\Omega)}^{2}+\|\theta\|_{L_{2}(\Omega)}^{2}\right) \leq E_{0}(t) \leq c_{2}\left(\|\boldsymbol{v}\|_{L_{2}(\Omega)}^{2}+\|\theta\|_{L_{2}(\Omega)}^{2}\right), \\
& E_{1}(t) \geq 2 \beta E_{0}(t), \quad \beta>0 \\
& E_{1}(t) \geq c_{3}\left(\|\boldsymbol{v}\|_{W_{2}^{1}(\Omega)}^{2}+\|\theta\|_{L_{2}(\Omega)}^{2}\right)
\end{aligned}
$$

provided $\gamma$ is sufficiently small. Hence,

$$
\frac{d E_{0}(t)}{d t}+2 \beta E_{0}(t)+E_{1}(t) \leq I(t)
$$

where $\beta>0$, and

$$
I(t)=2 \int_{\Omega}\left(\boldsymbol{f} \cdot(\boldsymbol{v}+\gamma \boldsymbol{V})+\frac{p^{\prime}(\bar{\rho})}{\bar{\rho}} h \theta\right) d x+2 \gamma \bar{\rho} \int_{\Omega} \boldsymbol{v} \cdot \boldsymbol{V}_{t} d x .
$$

It follows that

$$
e^{2 \beta t} E_{0}(t)+\int_{0}^{t} e^{2 \beta \tau} E_{1}(\tau) d \tau \leq E_{0}(0)+\int_{0}^{t} e^{2 \beta \tau}|I(\tau)| d \tau
$$

which leads to (2.3) (in view of (2.9)) after easy calculations.

In order to estimate higher order norms of $\boldsymbol{v}$ and $\theta$, we use a classical localization method. Let $\left\{\varphi_{k}(x)\right\}, k=1,2, \ldots, m$, be a partition of unity in $\Omega$ subordinate to the covering of $\Omega$ with the balls $\omega_{k}=\left\{\left|x-x_{k}\right| \leq d, x_{k} \in S\right\}, k=1, \ldots, m-1$, of a small radius $d$ plus a strictly interior subdomain $\omega_{m}$ of $\Omega$. We assume that every point of $\Omega$ can belong to not more than a fixed number $m_{1}$ of the $\omega_{k}$, moreover, the intersections $\omega_{k} \cap \omega_{m}$, $k<m$, contain balls $B_{k}$ of radius $\alpha d, \alpha<1$, and we are given smooth functions $\zeta_{k}(x)$ 
with $\operatorname{supp}_{k} \subset B_{k}, \int_{B_{k}} \zeta_{k}(x) d x=1$. Let $\theta_{k}(x, t)=\theta(x, t) \varphi_{k}(x), k \leq m, \omega_{k}^{\prime}=\omega_{k} \cap \Omega$, and let

$$
\begin{aligned}
& \vartheta_{k}(x, t)=\theta_{k}(x, t)-\zeta_{k}(x) \int_{\omega_{k}^{\prime}} \theta_{k}(y, t) d y, \quad k<m, \\
& \vartheta_{m}(x, t)=\theta_{m}(x, t)+\sum_{k=1}^{m-1} \zeta_{k}(x) \int_{\omega_{k}^{\prime}} \theta_{k}(y, t) d y .
\end{aligned}
$$

It is clear that $\operatorname{supp} \vartheta_{k} \subset \omega_{k}, \sum_{k=1}^{m} \vartheta_{k}(x, t)=\sum_{k=1}^{m} \theta_{k}=\theta(x, t)$, and

$$
\begin{aligned}
& \int_{\omega_{k}^{\prime}} \vartheta_{k}(x, t) d x=0, \quad k<m, \\
& \int_{\omega_{m}} \vartheta_{m}(x, t) d x=\int_{\omega_{m}} \theta_{m}(x, t) d x+\sum_{k=1}^{m-1} \int_{\omega_{k}^{\prime}} \theta_{k}(x, t) d x=0 .
\end{aligned}
$$

We also set $\boldsymbol{v}_{k}=\boldsymbol{v} \varphi_{k}$,

$$
\begin{aligned}
& \boldsymbol{w}_{k}(x, t)=\boldsymbol{v}_{k}(x, t)-\zeta_{k}(x) \int_{\omega_{k}^{\prime}} \boldsymbol{v}_{k}(x, t) d x, \quad k<m, \\
& \boldsymbol{w}_{m}(x, t)=\boldsymbol{v}_{m}(x, t)+\sum_{k=1}^{m-1} \zeta_{k}(x) \int_{\omega_{k}^{\prime}} \boldsymbol{v}_{k}(x, t) d x
\end{aligned}
$$

and compute the expressions

$$
\begin{aligned}
& \bar{\rho} \boldsymbol{w}_{k, t}-\nabla \cdot T\left(\boldsymbol{w}_{k}\right)+p^{\prime}(\bar{\rho}) \nabla \vartheta_{k}=\boldsymbol{f}_{k}+\mathfrak{f}_{k}, \\
& \vartheta_{k, t}+\bar{\rho} \nabla \cdot \boldsymbol{w}_{k}=h_{k}+\mathfrak{h}_{k},
\end{aligned}
$$

where

$$
\begin{aligned}
& \boldsymbol{f}_{k}=\boldsymbol{f} \varphi_{k}-\zeta_{k} \int_{\omega_{k}^{\prime}} \boldsymbol{f} \varphi_{k} d x, \quad h_{k}=h \varphi_{k}-\zeta_{k} \int_{\omega_{k}^{\prime}} h \varphi_{k} d x, \quad k<m, \\
& \boldsymbol{f}_{m}=\boldsymbol{f} \varphi_{m}+\sum_{k=1}^{m-1} \zeta_{k} \int_{\omega_{k}^{\prime}} \boldsymbol{f} \varphi_{k} d x, \quad h_{m}=h \varphi_{m}+\sum_{k=1}^{m-1} \zeta_{k} \int_{\omega_{k}^{\prime}} h \varphi_{k} d x,
\end{aligned}
$$

and the $\mathfrak{f}_{k}, \mathfrak{h}_{k}$ are functions containing the derivatives of the $\varphi_{k}, \zeta_{k}$ and, consequently, only lower order derivatives of $\boldsymbol{v}$ and $\theta$.

To obtain interior estimates for the derivatives of the solution, we consider system (2.14) with $k=m$. Since $\operatorname{supp} \boldsymbol{w}_{m}, \operatorname{supp} \vartheta_{m} \subset \omega_{m}$, we extend $\boldsymbol{w}_{m}$ and $\vartheta_{m}$ by zero to a certain cube $\mathfrak{Q}=\left\{\left|x_{j}-x_{j m}\right| \leq D\right\}, j=1,2,3$, containing $\omega_{m}$ (and outside $\mathfrak{Q}$ ). Inequality (2.10) for system (2.14) in $\mathfrak{Q}$ implies

$$
\begin{aligned}
& \int_{0}^{T} e^{2 \beta t}\left(\left\|\boldsymbol{w}_{m}(\cdot, t)\right\|_{W_{2}^{1}\left(\omega_{m}\right)}^{2}+\left\|\vartheta_{m}(\cdot, t)\right\|_{L_{2}\left(\omega_{m}\right)}^{2}\right) d t \\
& \quad \leq c\left(\left\|\boldsymbol{w}_{m}(\cdot, 0)\right\|_{L_{2}\left(\omega_{m}\right)}^{2}+\left\|\vartheta_{m}(\cdot, 0)\right\|_{L_{2}\left(\omega_{m}\right)}^{2}+\left|\int_{0}^{T} e^{2 \beta t} I_{m}(t) d t\right|\right)
\end{aligned}
$$

and, as a consequence,

$$
\begin{aligned}
\int_{0}^{T} e^{2 \beta t}\left(\left\|\boldsymbol{w}_{m}(\cdot, t)\right\|_{W_{2}^{1}\left(\omega_{m}\right)}^{2}\right. & \left.+\left\|\vartheta_{m}(\cdot, t)\right\|_{L_{2}\left(\omega_{m}\right)}^{2}\right) d t \\
\leq c\left(\left\|\boldsymbol{w}_{m}(\cdot, 0)\right\|_{L_{2}(\Omega)}^{2}\right. & +\left\|\vartheta_{m}(\cdot, 0)\right\|_{L_{2}(\Omega)}^{2} \\
& \left.+\int_{0}^{T} e^{2 \beta t}\left(\left\|\boldsymbol{f}_{m}+\mathfrak{f}_{m}\right\|_{L_{2}\left(\omega_{m}\right)}^{2}+\left\|h_{m}+\mathfrak{h}_{m}\right\|_{L_{2}\left(\omega_{m}\right)}^{2}\right) d t\right) .
\end{aligned}
$$


Let

$$
\Delta_{j}^{q}(z) f(x)=\sum_{k=0}^{q}(-1)^{q-k}\left(\begin{array}{l}
q \\
k
\end{array}\right) f\left(x+k \boldsymbol{e}_{j} z\right), \quad \boldsymbol{e}_{j}=\left(\delta_{i j}\right)_{i=1,2,3},
$$

be the $q$ th finite difference of $f(x)$ with respect to the variable $x_{j}, q>l+1$. Taking the finite differences of equations (2.14) with $k=m$, we obtain

$$
\begin{aligned}
\bar{\rho} \Delta_{j}^{q}(z) \boldsymbol{w}_{m, t}-\nabla \cdot T\left(\Delta_{j}^{q}(z) \boldsymbol{w}_{m}\right)+p^{\prime}(\bar{\rho}) \nabla \Delta_{j}^{q}(z) \vartheta_{m} & =\Delta_{j}^{q}(z)\left(\boldsymbol{f}_{m}+\mathfrak{f}_{m}\right), \\
\Delta_{j}^{q}(z) \vartheta_{m, t}+\bar{\rho} \nabla \cdot \Delta_{j}^{q}(z) \boldsymbol{w}_{m} & =\Delta_{j}^{q}(z)\left(h_{m}+\mathfrak{h}_{m}\right) .
\end{aligned}
$$

We assume that $0<z<z_{0}$ with $z_{0}$ so small (or $D$ so large) that $\operatorname{supp} \Delta_{j}^{q}(z) \boldsymbol{w}_{m}$, $\operatorname{supp} \Delta_{j}^{q}(z) \vartheta_{m}$ are contained in $\mathfrak{Q}$. Since $\int_{\mathfrak{Q}} \Delta_{j}^{q}(z) \vartheta_{m} d x=0$, we can introduce a vector field $\boldsymbol{W}_{m}(x, t)$ such that

$$
\begin{aligned}
\nabla \cdot \boldsymbol{W}_{m}(x, t) & =-\Delta_{j}^{q}(z) \theta_{m}(x, t), \quad x \in \mathfrak{Q}, \\
\left\|\boldsymbol{W}_{m}\right\|_{W_{2}^{1}(\mathfrak{Q})} & \leq c\left\|\Delta_{j}^{q}(z) \vartheta_{m}\right\|_{L_{2}(\mathfrak{Q})}, \\
\left\|\boldsymbol{W}_{m, t}\right\|_{L_{2}(\mathfrak{Q})} & \leq c\left(\left\|\Delta_{j}^{q}(z) \nabla \cdot \boldsymbol{w}_{m}\right\|_{L_{2}(\mathfrak{Q})}+\left\|\Delta_{j}^{q}(z)\left(h_{m}+\mathfrak{h}_{m}\right)\right\|_{L_{2}(\mathfrak{Q})}\right) .
\end{aligned}
$$

By Proposition 1 applied to system (2.18), we have

$$
\begin{aligned}
\int_{0}^{T} e^{2 \beta t}\left(\left\|\Delta_{j}^{q}(z) \boldsymbol{w}_{m}(\cdot, t)\right\|_{W_{2}^{1}(\mathfrak{Q})}^{2}+\left\|\Delta_{j}^{q}(z) \vartheta_{m}(\cdot, t)\right\|_{L_{2}(\mathfrak{Q})}^{2}\right) d t \\
\leq c\left(\left\|\boldsymbol{\Delta}_{j}^{q}(z) \boldsymbol{w}_{m}(\cdot, 0)\right\|_{L_{2}(\mathfrak{Q})}^{2}+\left\|\Delta_{j}^{q}(z) \vartheta_{m}(\cdot, 0)\right\|_{L_{2}(\mathfrak{Q})}^{2}\right. \\
+2 \mid \int_{0}^{T} e^{2 \beta t} d t \int_{\mathfrak{Q}}\left(\Delta_{j}^{q}(z)\left(\boldsymbol{f}_{m}+\mathfrak{f}_{m}\right) \cdot\left(\Delta_{j}^{q}(z) \boldsymbol{w}_{m}+\gamma \boldsymbol{W}_{m}(x, t)\right)\right. \\
\left.+\Delta_{j}^{q}(z)\left(h_{m}+\mathfrak{h}_{m}\right) \Delta_{j}^{q} \vartheta_{m}\right) d x \mid \\
\left.+2 \bar{\rho} \gamma\left|\int_{0}^{T} e^{2 \beta t} d t \int_{\mathfrak{Q}} \Delta_{j}^{q}(z) \boldsymbol{w}_{m} \cdot \boldsymbol{W}_{m, t}(x, t) d x\right|\right) .
\end{aligned}
$$

We make use of the identity

$$
\begin{aligned}
\int_{0}^{T} e^{2 \beta t} d t \int_{\mathfrak{Q}}\left(\Delta_{j}^{q}(z)\left(\boldsymbol{f}_{m}+\mathfrak{f}_{m}\right) \cdot\left(\Delta_{j}^{q}(z) \boldsymbol{w}_{m}+\gamma \boldsymbol{W}_{m}\right) d x\right. \\
\quad=-\int_{0}^{T} e^{2 \beta t} d t \int_{\mathfrak{Q}}\left(\Delta_{j}^{q-1}(z)\left(\boldsymbol{f}_{m}+\mathfrak{f}_{m}\right) \cdot\left(\Delta_{j}^{q+1} \boldsymbol{w}_{m}+\Delta_{j}(z) \boldsymbol{W}_{m}\right) d x\right.
\end{aligned}
$$

and estimate the penultimate integral in $(2.20)$ (denoted by $J_{m}$ ) as follows:

$$
\begin{array}{r}
\left|J_{m}\right| \leq c\left(\int _ { 0 } ^ { T } e ^ { 2 \beta t } \left(\left\|\Delta_{j}^{q-1}(z)\left(\boldsymbol{f}_{m}+\mathfrak{f}_{m}\right)\right\|_{L_{2}(\mathfrak{Q})}\left(\left\|\Delta_{j}^{q+1} \boldsymbol{w}_{m}\right\|_{L_{2}(\mathfrak{Q})}+|z|\left\|\nabla \boldsymbol{W}_{m}\right\|_{L_{2}(\mathfrak{Q})}\right) d t\right.\right. \\
\quad+\int_{0}^{T} e^{2 \beta t}\left(\left\|\Delta_{j}^{q}(z)\left(h_{m}+\mathfrak{h}_{m}\right)\right\|_{L_{2}(\mathfrak{Q})}\left\|\Delta_{j}^{q}(z) \vartheta_{m}\right\|_{L_{2}(\mathfrak{Q})} d t\right) \\
\leq c\left(\int_{0}^{T} e^{2 \beta t}\left\|\Delta_{j}^{q-1}(z)\left(\boldsymbol{f}_{m}+\mathfrak{f}_{m}\right)\right\|_{\left.L_{2} \mathfrak{Q}\right)}\left(\left\|\Delta_{j}^{q+1} \boldsymbol{w}_{m}\right\|_{L_{2}(\mathfrak{Q})}+|z|\left\|\Delta_{j}^{q}(z) \vartheta_{m}\right\|_{L_{2}(\mathfrak{Q})}\right) d t\right. \\
\left.+\int_{0}^{T} e^{2 \beta t}\left\|\Delta_{j}^{q}(z)\left(h_{m}+\mathfrak{h}_{m}\right)\right\|_{L_{2}(\mathfrak{Q})}\left\|\Delta_{j}^{q}(z) \vartheta_{m}\right\|_{L_{2}(\mathfrak{Q})} d t\right) .
\end{array}
$$

Next, we divide $(2.20)$ by $z^{1+2(l+1)}$ and integrate over $z \in\left(0, z_{0}\right)$. Since the norms

$$
\|u\|_{L_{2}(\mathfrak{Q})}+\sum_{j=1}^{3}\left(\int_{0}^{z_{0}}\left\|\Delta_{j}^{s}(z) u\right\|_{L_{2}(\mathfrak{Q})}^{2} \frac{d z}{z^{1+2 r}}\right)^{1 / 2}
$$


and $\|u\|_{W_{2}^{r}(\mathfrak{Q})}$ are equivalent for all $s>r>0$ (see [7]), our calculations lead to

$$
\begin{aligned}
\left\|e^{\beta t} \boldsymbol{w}_{m}\right\|_{W_{2}^{l+2,0}\left(\mathfrak{Q}_{T}\right)}^{2}+\left\|e^{\beta t} \vartheta_{m}\right\|_{W_{2}^{l+1,0}\left(\mathfrak{Q}_{T}\right)}^{2} & \\
\leq c\left(\left\|e^{\beta t}\left(\boldsymbol{f}_{m}+\mathfrak{f}_{m}\right)\right\|_{W_{2}^{l, l / 2}\left(\mathfrak{Q}_{T}\right)}^{2}\right. & +\left\|e^{\beta t}\left(h_{m}+\mathfrak{h}_{m}\right)\right\|_{W_{2}^{l+1,0}\left(\mathfrak{Q}_{T}\right)}^{2} \\
& \left.+\left\|\boldsymbol{w}_{m}(\cdot, 0)\right\|_{W_{2}^{l+1}(\mathfrak{Q})}^{2}+\left\|\vartheta_{m}(\cdot, 0)\right\|_{W_{2}^{l+1}(\mathfrak{Q})}^{2}\right) \\
& \equiv A_{m},
\end{aligned}
$$

where $\mathfrak{Q}_{T}=\mathfrak{Q} \times(0, T)$. Moreover, we have

$$
\begin{aligned}
& \left\|e^{\beta t} \vartheta_{m, t}\right\|_{W_{2}^{l+1,0}\left(\mathfrak{Q}_{T}\right)}^{2} \\
& \leq c\left(\left\|e^{\beta t} \nabla \cdot \boldsymbol{w}_{m}\right\|_{W_{2}^{l+1,0}\left(\mathfrak{Q}_{T}\right)}^{2}+\left\|e^{\beta t}\left(h_{m}+\mathfrak{h}_{m}\right)\right\|_{W_{2}^{l+1,0}\left(\mathfrak{Q}_{T}\right)}^{2}\right) \leq c A_{m}, \\
& \left\|e^{\beta t} \boldsymbol{w}_{m, t}\right\|_{W_{2}^{0, l / 2}\left(\mathfrak{Q}_{T}\right)}^{2} \leq c\left(\left\|e^{\beta t} D_{x}^{2} \boldsymbol{w}_{m}\right\|_{W_{2}^{0, l / 2}\left(\mathfrak{Q}_{T}\right)}^{2}+\left\|e^{\beta t} \nabla \vartheta_{m}\right\|_{W_{2}^{0, l / 2}\left(\mathfrak{Q}_{T}\right)}^{2}\right. \\
& \left.+\left\|e^{\beta t}\left(\boldsymbol{f}_{m}+\mathfrak{f}_{m}\right)\right\|_{W_{2}^{0, l / 2}\left(\mathfrak{Q}_{T}\right)}^{2}\right) .
\end{aligned}
$$

By the interpolation inequalities, the right-hand side in the last estimate is not less than

$$
\begin{aligned}
& \delta\left(\left\|e^{\beta t} \boldsymbol{w}_{m, t}\right\|_{W_{2}^{0, l / 2}\left(\mathfrak{Q}_{T}\right)}^{2}+\left\|e^{\beta t} \vartheta_{m, t}\right\|_{W_{2}^{l+1,0}\left(\mathfrak{Q}_{T}\right)}^{2}\right) \\
& +c(\delta)\left(\left\|e^{\beta t} \boldsymbol{w}_{m}\right\|_{W_{2}^{2+l, 0}\left(\mathfrak{Q}_{T}\right)}^{2}+\left\|e^{\beta t} \vartheta_{m}\right\|_{W_{2}^{l+1,0}\left(\mathfrak{Q}_{T}\right)}^{2}\right) \\
& \quad+c\left(\left\|e^{\beta t} \vartheta_{m, t}\right\|_{W_{2}^{l+1,0}\left(\mathfrak{Q}_{T}\right)}^{2}+\left\|e^{\beta t}\left(\boldsymbol{f}_{m}+\mathfrak{f}_{m}\right)\right\|_{W_{2}^{0, l / 2}\left(\mathfrak{Q}_{T}\right)}^{2}\right) .
\end{aligned}
$$

Collecting the estimates and choosing $\delta$ sufficiently small, we obtain the desired inequality

$$
\left\|e^{\beta t} \boldsymbol{w}_{m}\right\|_{W_{2}^{l+2, l / 2+1}\left(\mathfrak{Q}_{T}\right)}^{2}+\left\|e^{\beta t} \vartheta_{m}\right\|_{W_{2}^{l+1,0}\left(\mathfrak{Q}_{T}\right)}^{2}+\left\|e^{\beta t} \vartheta_{m, t}\right\|_{W_{2}^{l+1,0}\left(\mathfrak{Q}_{T}\right)}^{2} \leq c A_{m} .
$$

We pass to estimates for $\boldsymbol{v}$ and $\theta$ near the boundary; for this we consider equations (2.14) with $k<m$. Without loss of generality we may assume that the point $x_{k}$ coincides with the origin and the $x_{3}$-axis is directed along the interior normal $-\boldsymbol{n}\left(x_{k}\right)$. Let $x_{3}=$ $\phi\left(x^{\prime}\right), x^{\prime}=\left(x_{1}, x_{2}\right) \in K$, be the equation of the boundary $\mathrm{S}$ near the origin; by $K$ we mean the disk $|x| \leq d_{1}, d_{1}>d$.

We study system (2.14) assuming for simplicity that $S \in W_{2}^{2+l}$. We make the change of variables

$$
x_{1}=y_{1}, \quad x_{2}=y_{2}, \quad x_{3}=y_{3}+\phi\left(y^{\prime}\right),
$$

where $\phi \in W_{2}^{2+l}(K)$. The Jacobi matrix of this transformation is given by

$$
\mathfrak{L}=\left(\begin{array}{ccc}
1 & 0 & 0 \\
0 & 1 & 0 \\
\phi_{y_{1}} & \phi_{y_{2}} & 1
\end{array}\right)
$$

whence $\operatorname{det} \mathfrak{L}=1$, and $\mathfrak{L}^{-1}$ coincides with the co-factors matrix $\widehat{\mathfrak{L}}$. The transformation $(2.26)$ converts $\omega_{k} \cap \Omega$ (where the $\boldsymbol{w}_{k}$ and $\vartheta_{k}$ are given) into a certain $\widetilde{\omega}_{k} \subset \mathbb{R}_{+}^{3}=$ $\left\{x_{3}>0\right\}$. We extend $\boldsymbol{w}_{k}$ and $\vartheta_{k}$ by zero to a larger domain

$$
\mathfrak{Q}^{+}=\left\{0<y_{3}<2 d_{0}\right\}, \quad y^{\prime} \in \mathfrak{Q}^{\prime}=\left\{\left|y_{\alpha}\right|<d_{0}\right\}, \quad \alpha=1,2,
$$

where $d_{0}>d$. In the new coordinates $y$, system (2.14) takes the form

$$
\begin{aligned}
& \bar{\rho} \boldsymbol{w}_{k, t}-\tilde{\nabla} \cdot \tilde{T}\left(\boldsymbol{w}_{k}\right)+p^{\prime}(\bar{\rho}) \tilde{\nabla} \vartheta_{k}=\boldsymbol{f}_{k}+\mathfrak{f}_{k}, \\
& \vartheta_{k, t}+\bar{\rho} \tilde{\nabla} \cdot \boldsymbol{w}_{k}=h_{k}+\mathfrak{h}_{k}, \quad y \in \mathfrak{Q}^{+},
\end{aligned}
$$


where $\tilde{\nabla}=\mathfrak{L}^{-T} \nabla$ is the transformed gradient $\nabla_{x}, \widetilde{T}(\boldsymbol{u})=\mu \widetilde{S}(u)+\mu_{1} I \widetilde{\nabla} \cdot \boldsymbol{u}$ is the transformed stress tensor, and $\widetilde{S}(\boldsymbol{u})=\widetilde{\nabla} \boldsymbol{u}+(\widetilde{\nabla} \boldsymbol{u})^{T}$ is the transformed rate-of-strain tensor. We write system $(2.27)$, completed with a boundary condition on $\mathfrak{Q}^{\prime}$, in the form

$$
\begin{aligned}
& \bar{\rho} \boldsymbol{w}_{k, t}-\nabla \cdot T\left(\boldsymbol{w}_{k}\right)+p^{\prime}(\bar{\rho}) \nabla \vartheta_{k}=\boldsymbol{f}_{k}+\tilde{\mathfrak{f}}_{k}, \\
& \vartheta_{k, t}+\bar{\rho} \nabla \cdot \boldsymbol{w}_{k}=h_{k}+\tilde{\mathfrak{h}}_{k}, \quad y \in \mathfrak{Q}^{+}, \quad \boldsymbol{w}_{k}=0, \quad y^{\prime} \in \mathfrak{Q}^{\prime}, \quad y_{3}=0,
\end{aligned}
$$

where the functions

$$
\begin{aligned}
\tilde{\mathfrak{f}}_{k} & =\mathfrak{f}_{k}+\tilde{\nabla} \cdot \tilde{T}\left(\boldsymbol{w}_{k}\right)-\nabla \cdot T\left(\boldsymbol{w}_{k}\right)-p^{\prime}(\bar{\rho}) \widetilde{\nabla} \vartheta_{k}+p^{\prime}(\bar{\rho}) \nabla \vartheta_{k}, \\
\tilde{\mathfrak{h}}_{k} & =\mathfrak{h}_{k}-\bar{\rho} \tilde{\nabla} \cdot \boldsymbol{w}_{k}+\bar{\rho} \nabla \cdot \boldsymbol{w}_{k}
\end{aligned}
$$

involve also higher order derivatives of $\boldsymbol{w}_{k}$ and $\vartheta_{k}$ with small coefficients proportional to $\phi$ or to the derivatives of $\phi$. We note that

$$
\int_{\mathfrak{Q}^{+}} \vartheta_{k}(y, t) d y=0
$$

Remark. If $\phi \in W_{2}^{3 / 2+l}(K)$, then, instead of (2.26), the transformation

$$
x^{\prime}=y^{\prime}, \quad x_{3}=y_{3}+\phi^{*}(y)
$$

should be used, where $\phi^{*}$ is the extension of $\phi$ to $K \times\left(0,2 d_{0}\right)$ such that

$$
\begin{aligned}
& \phi^{*}(y)=\phi\left(y^{\prime}\right), \quad \frac{\partial \phi^{*}}{\partial y_{3}}=0, \quad y_{3}=0, \\
& \left\|\phi^{*}\right\|_{W_{2}^{l+2}\left(K \times\left(0,2 d_{0}\right)\right)} \leq c\|\phi\|_{W_{2}^{l+3 / 2}(K)}
\end{aligned}
$$

Since $\phi^{*}(0)=0$ and $\nabla \phi^{*}(0)=0$, we have

$$
\left|\phi^{*}(y)\right| \leq c|y|^{1+\alpha}, \quad\left|\nabla \phi^{*}(y)\right| \leq c|y|^{\alpha}, \quad \alpha=l-1 / 2 .
$$

The Jacobi matrix of the transformation (2.31) equals

$$
\mathfrak{L}=\left(\begin{array}{ccc}
1 & 0 & 0 \\
0 & 1 & 0 \\
\phi_{y_{1}}^{*} & \phi_{y_{2}}^{*} & 1+\phi_{y_{3}}^{*}
\end{array}\right),
$$

and $\operatorname{det} \mathfrak{L}=1+\phi_{y_{3}}^{*}$. Consequently,

$$
\int_{\widetilde{\omega}_{k}} \vartheta_{k}(y, t) d y=\int_{\widetilde{\omega}_{k}} \vartheta_{k}(y, t)(1-\operatorname{det} \mathfrak{L}) d y=-\int_{\widetilde{\omega}_{k}} \vartheta_{k}(y, t) \phi_{y_{3}}^{*} d y .
$$

In order to conserve condition (2.30), we can introduce a new function of the form

$$
\vartheta_{k}^{\prime}(y, t)=\vartheta_{k}(y, t)-\Sigma_{k}(y) \int_{\omega_{k}} \vartheta_{k}(x, t)(1-\operatorname{det} \mathfrak{L}) d x,
$$

where $\Sigma_{k}(y)$ is smooth, $\operatorname{supp} \Sigma_{k} \subset \mathfrak{Q}^{+}$, and $\int_{\text {supp } \Sigma_{k}} \Sigma_{k}(x) d x=1$. Clearly, the $\left(\boldsymbol{w}_{k}, \vartheta^{\prime}\right)$ satisfy a system of the type (2.28) with other $\widetilde{\mathfrak{f}}_{k}$ and $\widetilde{\mathfrak{h}}_{k}$, but possessing the same properties. Since the new system is treated in the same way as (2.28), we omit the details.

We proceed by studying equations (2.28). We follow the arguments in the proof of (2.23), keeping in mind that finite differences can be taken this time only with respect to tangential variables $y_{1}, y_{2}$. Therefore, instead of (2.23) we obtain

$$
\begin{aligned}
& \left\|e^{\beta t} \nabla \boldsymbol{w}_{k}\right\|_{W_{2, \tan }^{l+1,0}\left(\mathfrak{Q}_{T}^{+}\right)}^{2}+\left\|e^{\beta t} \boldsymbol{w}_{k}\right\|_{W_{2, \tan }^{l+1,0}\left(\mathfrak{Q}_{T}^{+}\right)}^{2}+\left\|e^{\beta t} \vartheta_{k}\right\|_{W_{2, \tan }^{l+1}\left(\mathfrak{Q}_{T}^{+}\right)}^{2} \\
& \leq c\left(\left\|\boldsymbol{w}_{k}(\cdot, 0)\right\|_{W_{2, \tan }^{l+1}\left(\mathfrak{Q}^{+}\right)}^{2}+\left\|\vartheta_{k}(\cdot, 0)\right\|_{W_{2, \tan }^{l+1}\left(\mathfrak{Q}^{+}\right)}^{2}\right. \\
& \left.\quad+\left\|e^{\beta t}\left(\boldsymbol{f}_{k}+\widetilde{\mathfrak{f}}_{k}\right)\right\|_{W_{2, \tan }^{l, 0}\left(\mathfrak{Q}_{T}^{+}\right)}^{2}+\left\|e^{\beta t}\left(h_{k}+\widetilde{\mathfrak{h}}_{k}\right)\right\|_{W_{2, \tan }^{l+1,0}\left(\mathfrak{Q}_{T}^{+}\right)}^{2}\right)
\end{aligned}
$$


where $\mathfrak{Q}_{T}^{+}=\mathfrak{Q}^{+} \times(0, T)$ and

$$
W_{2, \tan }^{r}\left(\mathfrak{Q}^{+}\right)=L_{2}\left(0,2 d_{0} ; W_{2}^{r}\left(\mathfrak{Q}^{\prime}\right)\right) .
$$

To estimate the derivatives of $\boldsymbol{w}_{k}, \vartheta_{k}$ with respect to $y_{3}$ and $t$, we rewrite (2.28) leaving on the left-hand side only these derivatives and carring all the remaining terms over to the right. In this way we arrive at a problem that can be viewed as one-dimensional in the interval $\left(0,2 d_{0}\right)$, namely,

$$
\begin{gathered}
\left\{\begin{array}{l}
\bar{\rho} w_{\alpha, t}-\mu \frac{\partial^{2} w_{\alpha}}{\partial y_{3}^{2}}=f_{\alpha}+\tilde{\mathfrak{f}}_{\alpha}+\mathfrak{f}_{\alpha}^{\prime} \equiv g_{\alpha}, \quad \alpha=1,2, \\
\left.w_{\alpha}\right|_{t=0}=w_{\alpha, 0},\left.\quad w_{\alpha}\right|_{y_{3}=0,2 d_{0}}=0,
\end{array}\right. \\
\left\{\begin{array}{l}
\bar{\rho} w_{3, t}-\left(2 \mu+\mu_{1}\right) \frac{\partial^{2} w_{3}}{\partial y_{3}^{2}}+p^{\prime}(\bar{\rho}) \frac{\partial \vartheta}{\partial y_{3}}=f_{3}+\tilde{\mathfrak{f}}_{3}+\mathfrak{f}_{3}^{\prime} \equiv g_{3}, \\
\vartheta_{t}+\bar{\rho} \frac{\partial w_{3}}{\partial y_{3}}=h+\tilde{\mathfrak{h}}+\mathfrak{h}^{\prime} \equiv e, \\
\left.w_{3}\right|_{y_{3}=0}=\left.w_{3}\right|_{y_{3}=2 d_{0}}=0,\left.\quad w_{3}\right|_{t=0}=w_{3,0},\left.\quad \vartheta\right|_{t=0}=\vartheta_{0},
\end{array}\right.
\end{gathered}
$$

(we have omitted the index $k$ for a moment). The functions $\mathfrak{f}_{i}^{\prime}, \mathfrak{h}^{\prime}, i=1,2,3$, stem from the transposed terms estimated in (2.34).

We consider problem (2.36) for $w_{3}$ and $\vartheta$. We start by reducing (2.36) to a similar problem with homogeneous initial data. We set $w_{3}^{(1)}(x, t)=u(x, t) \chi(t)$, where $\chi(t)$ is a smooth cut-off function equal to 1 for $t \in[0,1]$ and to zero for $t \geq 2$, and we require that

$$
\begin{aligned}
& u(x, 0)=w_{3.0}(x), \quad u(x, t)=0, \quad x_{3}=0,2 d_{0}, \\
& \|u\|_{W_{2}^{2+l, 1+l / 2}\left(\left(0,2 d_{0}\right) \times(0,2)\right)} \leq c\left\|w_{3,0}\right\|_{W_{2}^{1+l}\left(0,2 d_{0}\right)}
\end{aligned}
$$

for arbitrary fixed $x^{\prime} \in \mathfrak{Q}^{\prime}$; the function $w_{3}^{(1)}$ satisfies a similar inequality. The function $\vartheta^{(1)}$ is defined by $\vartheta^{(1)}(x, t)=\vartheta_{0}(x) \chi(t)$; it is clear that

$$
\left\|\vartheta^{(1)}\right\|_{W_{2}^{1+l, 0}\left(\left(0,2 d_{0}\right) \times(0,2)\right)}+\left\|\vartheta_{t}^{(1)}\right\|_{W_{2}^{1+l, 0}\left(\left(0,2 d_{0}\right) \times(0,2)\right)} \leq c\left\|\vartheta_{0}\right\|_{W_{2}^{1+l}\left(0,2 d_{0}\right)} .
$$

For $w_{3}^{(2)}=w_{3}-w_{3}^{(1)}$ and $\vartheta^{(2)}=\vartheta-\vartheta^{(1)}$ we obtain the problem

$$
\left\{\begin{array}{l}
\bar{\rho} w_{3, t}^{(2)}-\left(2 \mu+\mu_{1}\right) \frac{\partial^{2} w_{3}^{(2)}}{\partial y_{3}^{2}}+p^{\prime}(\bar{\rho}) \frac{\partial \vartheta^{(2)}}{\partial y_{3}}=g_{3}^{(1)} \\
\vartheta_{t}^{(2)}+\bar{\rho} \frac{\partial w_{3}^{(2)}}{\partial y_{3}}=e^{(1)}, \quad y_{3} \in\left(0,2 d_{0}\right), \\
w_{3}=0, \quad y_{3}=0,2 d_{0},\left.\quad w_{3}\right|_{t=0}=0,\left.\quad \vartheta\right|_{t=0}=0
\end{array}\right.
$$

where

$$
\begin{aligned}
g_{3}^{(1)} & =g_{3}-\bar{\rho} w_{3, t}^{(1)}+\left(2 \mu+\mu_{1}\right) \frac{\partial^{2} w_{3}^{(1)}}{\partial y_{3}^{2}}-p^{\prime}(\bar{\rho}) \frac{\partial \vartheta^{(1)}}{\partial y_{3}}, \\
e^{(1)} & =e-\vartheta_{t}^{(1)}-\bar{\rho} \frac{\partial w_{3}^{(1)}}{\partial y_{3}} .
\end{aligned}
$$

We extend all the functions in (2.39) by zero to the domain $t<0$ and apply the Laplace transformation defined by a standard formula

$$
\widetilde{u}(s)=\int_{0}^{\infty} e^{-s t} u(t) d t .
$$

For the transformed functions $\widetilde{w}_{3}^{(2)}$ and $\widetilde{\vartheta}^{(2)}$ we obtain the equations

$$
\left\{\begin{array}{l}
\bar{\rho} s \widetilde{w}_{3}^{(2)}-\left(2 \mu+\mu_{1}\right) \frac{d^{2} \widetilde{w}_{3}^{(2)}}{d y_{3}^{2}}+p^{\prime}(\bar{\rho}) \frac{d \widetilde{\vartheta}^{(2)}}{d y_{3}}=\widetilde{g}_{3}^{(1)}, \\
s \widetilde{\vartheta}^{(2)}+\bar{\rho} \frac{d w_{3}^{(2)}}{d y_{3}}=\widetilde{e}^{(1)} \\
\widetilde{w}_{3}^{(2)}=0, \quad y_{3}=0,2 d_{0} .
\end{array}\right.
$$


Upon eliminating $\widetilde{\vartheta}^{(2)}$, we arrive at the Sturm-Liouville problem

$$
\left\{\begin{array}{l}
\bar{\rho} s \widetilde{w}_{3}^{(2)}-\left(2 \mu+\mu_{1}+\frac{\bar{\rho} p^{\prime}(\bar{\rho})}{s}\right) \frac{d^{2} \widetilde{w}_{3}^{(2)}}{d y_{3}^{2}}=\widetilde{g}_{3}^{(1)}-\frac{p^{\prime}(\bar{\rho})}{s} \frac{d \widetilde{e}^{(1)}}{d y_{3}}, \\
\widetilde{w}_{3}^{(2)}=0, \quad y_{3}=0,2 d_{0}
\end{array}\right.
$$

or

$$
\left\{\begin{array}{l}
R(s) \widetilde{w}_{3}^{(2)}-\frac{d^{2} \widetilde{w}_{3}^{(2)}}{d y_{3}^{2}}=\widetilde{G} \\
\widetilde{w}_{3}^{(2)}=0, \quad y_{3}=0,2 d_{0}
\end{array}\right.
$$

where

$$
\begin{aligned}
R(s) & =\frac{\bar{\rho} s^{2}}{a s+b}, \quad a=2 \mu+\mu_{1}>0, \quad b=\bar{\rho} p^{\prime}(\bar{\rho})>0, \\
\widetilde{G} & =\frac{s}{a s+b} \widetilde{g}_{3}^{(1)}-\frac{p^{\prime}(\bar{\rho})}{a s+b} \frac{d \widetilde{e}^{(1)}}{d y_{3}} .
\end{aligned}
$$

The solution of (2.41) is given by the well-known formula (see [8])

$$
\widetilde{w}_{3}^{(2)}\left(y_{3}\right)=C^{-1}\left(\int_{y_{3}}^{2 d_{0}} z_{2}(\eta) z_{1}\left(y_{3}\right) \widetilde{G}(\eta) d \eta+\int_{0}^{y_{3}} z_{2}\left(y_{3}\right) z_{1}(\eta) \widetilde{G}(\eta) d \eta\right),
$$

where

$$
z_{1}(\eta)=e^{r \eta}-e^{-r \eta}, \quad z_{2}=e^{\left(2 d_{0}-\eta\right) r}-e^{-\left(2 d_{0}-\eta\right) r}, \quad r=\sqrt{R(s)}=\sqrt{\bar{\rho}} \frac{s}{\sqrt{a s+b}},
$$

are solutions of the homogeneous equation $R(s) z-z^{\prime \prime}=0$ vanishing for $\eta=0$ and $\eta=2 d_{0}$, respectively, while $C$ is the Wronskian of $z_{1}$ and $z_{2}$ :

$$
C=z_{2}^{\prime}(\eta) z_{1}(\eta)-z_{1}^{\prime}(\eta) z_{2}(\eta)=2 r\left(e^{-2 d_{0} r}-e^{2 d_{0} r}\right) .
$$

Since $r(s)$ is an analytic function for $\operatorname{Re} s>-b / a$, we can fix $\operatorname{Re} s=s_{1} \in(-b / a, 0)$. It is not hard to verify that under this condition the function (2.43) satisfies the inequality

$$
|s|^{2}\left\|\widetilde{w}_{3}^{(2)}\right\|_{L_{2}\left(0,2 d_{0}\right)}^{2} \leq c\left(s_{1}\right)\|\widetilde{G}\|_{L_{2}\left(0,2 d_{0}\right)}^{2} .
$$

We multiply (2.44) by $|s|^{l}$ and integrate over the line Re $s=s_{1}$. This leads to an inequality equivalent to

$$
\left\|e^{\beta t} w_{3}^{(2)}\right\|_{W_{2}^{0,1+l / 2}\left(\left(0,2 d_{0}\right) \times(0, \infty)\right)}^{2} \leq c \| e^{\beta t} G_{W_{2}^{0, l / 2}\left(\left(0,2 d_{0}\right) \times(0, \infty)\right)}^{2}
$$

with $\beta=-s_{1}>0$. Integrating (2.45) over $\mathfrak{Q}^{\prime}$, we obtain

$$
\left\|e^{\beta t} w_{3}^{(2)}\right\|_{W_{2}^{0,1+l / 2}\left(\mathfrak{Q}_{\infty}^{+}\right)}^{2} \leq c\left\|e^{\beta t} G\right\|_{W_{2}^{0, l / 2}\left(\mathfrak{Q}_{\infty}^{+}\right)}^{2} .
$$

Next, we estimate the $W_{2}^{2+l}\left(0,2 d_{0}\right)$-norm of $\widetilde{w}_{3}^{(2)}$ by using equation (2.41). We have

$$
\left\|D_{y_{3}}^{2} \widetilde{w}_{3}^{(2)}\right\|_{W_{2}^{l}\left(0,2 d_{0}\right)} \leq c\left(|R(s)|\left\|\widetilde{w}_{3}^{(2)}\right\|_{W_{2}^{l}\left(0,2 d_{0}\right)}+\|\widetilde{G}\|_{W_{2}^{l}\left(0,2 d_{0}\right)}\right),
$$

which implies, by the interpolation inequality,

$$
\begin{array}{ll}
\left\|e^{\beta t} w_{3}^{(2)}\right\|_{W_{2}^{2+l, 0}\left(\left(0,2 d_{0}\right) \times(0, \infty)\right)} & \\
\leq c\left(\left\|e^{\beta t} D_{t} w_{3}^{(2)}\right\|_{W_{2}^{l, 0}\left(\left(0,2 d_{0}\right) \times(0, \infty)\right)}+\left\|e^{\beta t} G\right\|_{W_{2}^{l, 0}\left(\left(0,2 d_{0}\right) \times(0, \infty)\right)}\right) \\
\leq \delta\left\|e^{\beta t} w_{3}^{(2)}\right\|_{W_{2}^{2+l, 0}\left(\left(0,2 d_{0}\right) \times(0, \infty)\right)}+c(\delta)\left\|e^{\beta t} w_{3}^{(2)}\right\|_{W_{2}^{0,1+l / 2}\left(\left(0,2 d_{0}\right) \times(0, \infty)\right)} \\
\quad+c\left\|e^{\beta t} G\right\|_{W_{2}^{l, l / 2}\left(\left(0,2 d_{0}\right) \times(0, \infty)\right)} .
\end{array}
$$

Choosing $\delta$ sufficiently small and taking (2.45) into account, we obtain

$$
\left\|e^{\beta t} w_{3}^{(2)}\right\|_{W_{2}^{2+l, 1+l / 2}\left(\left(0,2 d_{0}\right) \times(0, \infty)\right)}^{2} \leq c\left\|e^{\beta t} G\right\|_{W_{2}^{l, l / 2}\left(\left(0,2 d_{0}\right) \times(0, \infty)\right)}^{2} .
$$


Finally, we use (2.37), (2.38), and (2.45), to get

$$
\begin{aligned}
&\left\|e^{\beta t} w_{3}\right\|_{L_{2}\left(\mathfrak{Q}^{\prime}, W_{2}^{2+l, 1+l / 2}\left(\left(0,2 d_{0}\right) \times(0, \infty)\right)\right)}^{2}+\left\|e^{\beta t} \vartheta\right\|_{L_{2}\left(\mathfrak{Q}^{\prime}, W_{2}^{1+l, 0}\left(\left(0,2 d_{0}\right) \times(0, \infty)\right)\right)}^{2} \\
& \quad+\left\|e^{\beta t} \vartheta_{t}\right\|_{L_{2}\left(\mathfrak{Q}^{\prime}, W_{2}^{1+l, 0}\left(\left(0,2 d_{0}\right) \times(0, \infty)\right)\right)}^{2} \\
& \leq c\left(\left\|e^{\beta t} g_{3}\right\|_{L_{2}\left(\mathfrak{Q}^{\prime}, W_{2}^{l, l / 2}\left(\left(0,2 d_{0}\right) \times(0, \infty)\right)\right)}^{2}+\left\|e^{\beta t} \frac{\partial e}{\partial y_{3}}\right\|_{L_{2}\left(\mathfrak{Q}^{\prime}, W_{2}^{l, 0}\left(\left(0,2 d_{0}\right) \times(0, \infty)\right)\right)}^{2}\right. \\
&\left.\quad+\left\|w_{3,0}\right\|_{L_{2}\left(\mathfrak{Q}^{\prime}, W_{2}^{l+1}\left(0,2 d_{0}\right)\right)}^{2}+\left\|\vartheta_{0}\right\|_{L_{2}\left(\mathfrak{Q}^{\prime}, W_{2}^{l+1}\left(0,2 d_{0}\right)\right)}^{2}\right) .
\end{aligned}
$$

The functions $w_{\alpha}$ can be estimated by similar (in fact, more elementary) calculations, so that we have

$$
\begin{aligned}
\left.\left\|e^{\beta t} \boldsymbol{w}\right\|_{L_{2}\left(\mathfrak{Q}^{\prime}, W_{2}^{2}\right.}^{2+l, 1+l / 2}\left(\left(0,2 d_{0}\right) \times(0, \infty)\right)\right) & +\left\|e^{\beta t} \vartheta\right\|_{L_{2}\left(\mathfrak{Q}^{\prime}, W_{2}^{1+l, 0}\left(\left(0,2 d_{0}\right) \times(0, \infty)\right)\right)}^{2} \\
& +\left\|e^{\beta t} \vartheta_{t}\right\|_{L_{2}\left(\mathfrak{Q}^{\prime}, W_{2}^{1+l, 0}\left(\left(0,2 d_{0}\right) \times(0, \infty)\right)\right)}^{2} \\
\leq & c\left(\left\|e^{\beta t} \boldsymbol{g}\right\|_{L_{2}\left(\mathfrak{Q}^{\prime}, W_{2}^{l, l / 2}\left(\left(0,2 d_{0}\right) \times(0, \infty)\right)\right)}^{2}+\left\|e^{\beta t} \nabla e\right\|_{L_{2}\left(\mathfrak{Q}^{\prime}, W_{2}^{l, 0}\left(\left(0,2 d_{0}\right) \times(0, \infty)\right)\right)}^{2}\right. \\
& \left.+\left\|\boldsymbol{w}_{0}\right\|_{L_{2}\left(\mathfrak{Q}^{\prime}, W_{2}^{l+1}\left(0,2 d_{0}\right)\right)}^{2}+\left\|\vartheta_{0}\right\|_{L_{2}\left(\mathfrak{Q}^{\prime}, W_{2}^{l+1}\left(0,2 d_{0}\right)\right)}^{2}\right) .
\end{aligned}
$$

Since the interval $(0, \infty)$ in $(2.45)-(2.50)$ can be replaced by $(0, T)$, as in parabolic problems [20], inequalities (2.50) and (2.34) imply

$$
\begin{aligned}
& \left\|e^{2 \beta t} \boldsymbol{w}_{k}\right\|_{W_{2}^{l+2,1+l / 2}\left(\mathfrak{Q}_{T}^{+}\right)}^{2}+\left\|e^{2 \beta t} \vartheta_{k}\right\|_{W_{2}^{l+1,0}\left(\mathfrak{Q}_{T}^{+}\right)}^{2}+\left\|e^{2 \beta t} \vartheta_{k, t}\right\|_{W_{2}^{l+1,0}\left(\mathfrak{Q}_{T}^{+}\right)}^{2} \\
& \leq c\left(\left\|\boldsymbol{g}_{k}\right\|_{W_{2}^{l, l / 2}\left(\mathfrak{Q}_{T}^{+}\right)}^{2}+\left\|e_{k}\right\|_{W_{2}^{l+1,0}\left(\mathfrak{Q}_{T}^{+}\right)}^{2}+\left\|\boldsymbol{w}_{k}(\cdot, 0)\right\|_{W_{2}^{l+1}\left(\mathfrak{Q}^{+}\right)}^{2}+\left\|\vartheta_{k}(\cdot, 0)\right\|_{W_{2}^{l+1}\left(\mathfrak{Q}^{+}\right)}^{2}\right),
\end{aligned}
$$

where

$$
\boldsymbol{g}_{k}=\boldsymbol{f}_{k}+\tilde{\mathfrak{f}}_{k}+\mathfrak{f}_{k}^{\prime}, \quad e_{k}=h_{k}+\tilde{\mathfrak{h}}_{k}+\mathfrak{h}_{k}^{\prime} .
$$

We recall that the functions $\mathfrak{f}_{k}^{\prime}$ and $\mathfrak{h}_{k}^{\prime}$ are linear combinations of the derivatives of $\boldsymbol{w}_{k}$ and $\vartheta_{k}$, except for $\boldsymbol{w}_{k, t}, \vartheta_{k, t} D_{y_{3}}^{2} \boldsymbol{w}_{k}, D_{y_{3}} \vartheta_{k}$. They can be estimated by interpolation inequalities, which yields

$$
\begin{aligned}
\left\|e^{\beta t} \mathfrak{f}_{k}^{\prime}\right\|_{L_{2}\left(\mathfrak{Q}^{\prime}, W_{2}^{l, l / 2}\left(\left(0,2 d_{0}\right) \times(0, T)\right)\right.}^{2} & +\left\|e^{\beta t} \mathfrak{h}_{k}^{\prime}\right\|_{L_{2}\left(\mathfrak{Q}^{\prime}, W_{2}^{l+l, 0}\left(\left(0,2 d_{0}\right) \times(0, T)\right)\right)}^{2} \\
\leq \delta\left(\left\|e^{\beta t} \boldsymbol{w}_{k}\right\|_{W_{2}^{l+2,1+l / 2}\left(\mathfrak{Q}_{T}^{+}\right)}^{2}\right. & \left.+\left\|e^{\beta t} \vartheta_{k}\right\|_{W_{2}^{l+1,0}\left(\mathfrak{Q}_{T}^{+}\right)}^{2}+\left\|e^{\beta t} \vartheta_{k, t}\right\|_{W_{2}^{l+1,0}\left(\mathfrak{Q}_{T}^{+}\right)}^{2}\right) \\
& +c(\delta)\left(\left\|e^{\beta t} \boldsymbol{w}_{k}\right\|_{W_{2, \tan }^{l+2,0}\left(\mathfrak{Q}_{T}^{+}\right)}^{2}+\left\|e^{\beta t} \vartheta_{k}\right\|_{W_{2, \tan }^{l+1,0}\left(\mathfrak{Q}_{T}^{+}\right)}^{2}\right) .
\end{aligned}
$$

Hence, for small $\delta$ we have

$$
\begin{aligned}
&\left\|e^{\beta t} \boldsymbol{w}_{k}\right\|_{W_{2}^{l+2,1+l / 2}\left(\mathfrak{Q}_{T}^{+}\right)}^{2}+\left\|e^{\beta t} \vartheta_{k}\right\|_{W_{2}^{l+1,0}\left(\mathfrak{Q}_{T}^{+}\right)}^{2}+\left\|e^{\beta t} \vartheta_{k, t}\right\|_{W_{2}^{l+1,0}\left(\mathfrak{Q}_{T}^{+}\right)}^{2} \\
& \leq c\left(\left\|e^{\beta t}\left(\boldsymbol{f}_{k}+\tilde{\mathfrak{f}}_{k}\right)\right\|_{W_{2}^{l, l / 2}\left(\mathfrak{Q}_{T}^{+}\right)}^{2}\right.+\left\|e^{\beta t}\left(h_{k}+\widetilde{\mathfrak{h}}_{k}\right)\right\|_{W_{2}^{l+1,0}\left(\mathfrak{Q}_{T}^{+}\right)}^{2} \\
&\left.+\left\|\boldsymbol{w}_{k}(\cdot, 0)\right\|_{W_{2}^{l+1}\left(\mathfrak{Q}^{+}\right)}^{2}+\left\|\vartheta_{k}(\cdot, 0)\right\|_{W_{2}^{l+1}\left(\mathfrak{Q}^{+}\right)}^{2}\right),
\end{aligned}
$$

Since $\boldsymbol{v}=\sum_{k=1}^{m} \boldsymbol{v} \varphi_{k}$, the norms $\left\|e^{\beta t} \boldsymbol{v}\right\|_{W_{2}^{2+l, 1+l / 2}\left(Q_{T}\right)}^{2}$ and

$$
\sum_{k=1}^{m}\left\|e^{\beta t} \boldsymbol{v}_{k}\right\|_{W_{2}^{2+l, 1+l / 2}\left(\omega_{k} \times(0, T)\right)}^{2}
$$

are equivalent. The differences $\boldsymbol{w}_{k}-\boldsymbol{v}_{k}=-\zeta_{k} \int_{\omega_{k}} \boldsymbol{v}_{k} d x, k<m$, involve no higher order terms and can be estimated by interpolation inequalities, like $\mathfrak{f}_{k}$ and $\mathfrak{h}_{k}$. The same is 
true for $\vartheta_{k}-\theta_{k}$. Hence,

$$
\begin{aligned}
\sum_{k=1}^{m}\left\|e^{\beta t} \boldsymbol{v}_{k}\right\|_{W_{2}^{2+l, 1+l / 2}\left(\omega_{k} \times(0, T)\right)}^{2}+\sum_{k=1}^{m}\left\|e^{\beta t} \theta_{k}\right\|_{W_{2}^{1+l, 0}\left(\omega_{k} \times(0, T)\right)}^{2} & \\
& +\sum_{k=1}^{m}\left\|e^{\beta t} \theta_{k, t}\right\|_{W_{2}^{1+l, 0}\left(\omega_{k} \times(0, T)\right)}^{2} \\
\leq & c\left(\left\|\boldsymbol{v}_{0}\right\|_{W_{2}^{l+1}(\Omega)}^{2}+\left\|\theta_{0}\right\|_{W_{2}^{l+1}(\Omega)}^{2}+\left\|e^{\beta t} \boldsymbol{f}\right\|_{W_{2}^{l, l / 2}\left(Q_{T}\right)}^{2}+\left\|e^{\beta t} h\right\|_{W_{2}^{l+1,0}\left(Q_{\infty}\right)}^{2}\right) \\
& +\delta\left(\left\|e^{\beta t} \boldsymbol{v}\right\|_{W_{2}^{2+l, 1+l / 2}\left(Q_{\infty}\right)}^{2}+\left\|e^{\beta t} \theta_{t}\right\|_{W_{2}^{1+l, 0}\left(Q_{T}\right)}^{2}+\left\|e^{\beta t} \theta\right\|_{W_{2}^{1+l, 0}\left(Q_{T}\right)}^{2}\right) \\
& +c(\delta)\left(\left\|e^{\beta t} \boldsymbol{v}\right\|_{L_{2}\left(Q_{T}\right)}^{2}+\left\|e^{\beta t} \theta\right\|_{L_{2}\left(Q_{T}\right)}^{2}\right) .
\end{aligned}
$$

Since the weighted $L_{2}$-norms of $\boldsymbol{v}$ and $\theta$ were already estimated in (2.3), inequalities (2.3) and (2.54) imply (2.2) if $\delta$ is chosen small. This completes the proof of inequality $(2.2)$.

As for the solvability of problem (1.7) in an arbitrary finite time interval $(0, T)$, it suffices to prove this in the case of zero initial data. Eliminating $\theta$, we can write (1.7) in the form

$$
\left\{\begin{array}{l}
\bar{\rho} \boldsymbol{v}_{t}-\nabla \cdot T(\boldsymbol{v})=\boldsymbol{f}+p^{\prime}(\bar{\rho}) \int_{0}^{t} \nabla(\nabla \cdot \boldsymbol{v}) d \tau+p^{\prime}(\bar{\rho}) \bar{\rho} \int_{0}^{t} \nabla h d \tau, \quad x \in \Omega \\
\boldsymbol{v}(x, t)=0, \quad x \in S, \quad t>0 \\
\boldsymbol{v}(x, 0)=0, \quad x \in \Omega
\end{array}\right.
$$

which shows that the solvability of (1.7) follows from that of a similar problem for the Lamé system

$$
\left\{\begin{array}{l}
\bar{\rho} \boldsymbol{v}_{t}-\nabla \cdot T(\boldsymbol{v})=\boldsymbol{f} \\
\boldsymbol{v}(x, t)=0, \quad x \in S, \quad t>0 \\
\boldsymbol{v}(x, 0)=0, \quad x \in \Omega .
\end{array}\right.
$$

The latter solvability can be established in the same way (and even much easier) as in 12 for the case of the more complicated boundary conditions

$$
\left(T(\boldsymbol{v}) \boldsymbol{n}_{0}\right)_{\tau}=0, \quad \boldsymbol{n}_{0} \cdot T(\boldsymbol{v}) \boldsymbol{n}_{0}-\sigma \boldsymbol{n}_{0} \int_{0}^{t} \Delta_{0} \boldsymbol{v} d \tau=0,
$$

where $\Delta_{0}$ is the Laplace-Beltrami operator on $\Gamma_{0}$.

In conclusion, we consider problem (1.7) with initial data that do not obey (2.1).

Theorem 3. For arbitrary $\boldsymbol{v}_{0} \in W_{2}^{l+1}(\Omega), \rho_{0} \in W_{2}^{l+1}(\Omega), \boldsymbol{f} \in W_{2}^{l, l / 2}\left(Q_{T}\right)$, and $h \in$ $W_{2}^{l+1,0}\left(Q_{T}\right)$ satisfying $\boldsymbol{v}_{0}(x)=0, x \in S$, problem (1.7) has a unique solution $\boldsymbol{v} \in$ $W_{2}^{2+l, 1+l / 2}\left(Q_{T}\right), \theta, \theta_{t} \in W_{2}^{l+1,0}\left(Q_{T}\right), T<\infty$, and

$$
\begin{aligned}
& \|\boldsymbol{v}\|_{W_{2}^{l+2, l / 2+1}\left(Q_{T}\right)}+\|\theta\|_{W_{2}^{l+1,0}\left(Q_{T}\right)}+\left\|\theta_{t}\right\|_{W_{2}^{l+1,0}\left(Q_{T}\right)} \\
& \quad \leq c(T)\left(\left\|\boldsymbol{v}_{0}\right\|_{W_{2}^{l+1}(\Omega)}+\left\|\theta_{0}\right\|_{W_{2}^{l+1}(\Omega)}+\|\boldsymbol{f}\|_{W_{2}^{l, l / 2}\left(Q_{T}\right)}+\|h\|_{W_{2}^{l+1,0}\left(Q_{T}\right)}\right) .
\end{aligned}
$$

This theorem follows from Theorem 2, because the functions $\boldsymbol{v}$ and

$$
\theta^{\prime}=\theta-\frac{1}{|\Omega|}\left(\int_{\Omega} \theta_{0} d x+\int_{0}^{t} \int_{\Omega} h d x d \tau\right)=\theta-\frac{1}{|\Omega|} \int_{\Omega} \theta d x
$$

solve problem (1.7) with the initial data

$$
\left(\boldsymbol{v}_{0}, \theta_{0}^{\prime}=\theta_{0}-\frac{1}{|\Omega|} \int_{\Omega} \theta_{0} d x\right)
$$


and with $h^{\prime}=h-|\Omega|^{-1} \int_{\Omega} h d x$ in place of $h$ on the right-hand side. In this problem conditions (2.1) are satisfied.

\section{§3. NONLINEAR PROBlem}

In this section, we outline the proof of Theorem 1. It is based on Theorem 2 and on the following estimates of nonlinear terms.

Proposition 2. Let $p \in C^{2}(\bar{\rho} / 2,3 \bar{\rho} / 2), \boldsymbol{U}(\xi, t)=\int_{0}^{t} \boldsymbol{u}(\xi, \tau) d \tau$. If

$$
\begin{aligned}
& \sup _{t<T}\|\boldsymbol{U}(\cdot, t)\|_{W_{2}^{2+l}(\Omega)} \leq \delta_{1}, \quad \delta_{1}>0, \\
& \sup _{Q_{T}}|\theta(x, t)| \leq \bar{\rho} / 2,
\end{aligned}
$$

then

$$
Z(T) \leq c\left(\delta_{1} Y(T)+Y^{2}(T)\right)
$$

where $T>1$ and

$$
\begin{aligned}
Z(T) \equiv & Z(\boldsymbol{u}, q)=\left\|\boldsymbol{l}_{1}(\boldsymbol{u}, \theta)\right\|_{W_{2}^{l, l / 2}\left(Q_{T}\right)}+\left\|l_{2}(\boldsymbol{u}, \theta)\right\|_{W_{2}^{l+1,0}\left(Q_{T}\right)}, \\
Y(T) \equiv & Y(\boldsymbol{u}, q)=\|\boldsymbol{u}\|_{W_{2}^{2+l, 1+l / 2}\left(Q_{T}\right)}+\|\theta\|_{W_{2}^{l+1,0}\left(Q_{T}\right)}+\left\|\theta_{t}\right\|_{W_{2}^{l+1,0}\left(Q_{T}\right)}, \\
& l \in(1 / 2,1) .
\end{aligned}
$$

Proof. We use the auxiliary inequalities

$$
\begin{aligned}
\|f g\|_{W_{2}^{l}(\Omega)} & \leq c\|f\|_{W_{2}^{l}(\Omega)}\|g\|_{W_{2}^{l+1}(\Omega)}, \\
\|f g\|_{L_{2}(\Omega)} & \leq c\|f\|_{L_{p}(\Omega)}\|g\|_{L_{q}(\Omega)} \leq c\|f\|_{W_{2}^{l}(\Omega)}\|g\|_{W_{2}^{1}(\Omega)},
\end{aligned}
$$

where $1 / p+1 / q=1 / 2, l-3 / 2+3 / p=0$. The condition $l>1 / 2$ implies that $1-3 / 2+3 / q>$ 0 . Setting

$$
\|u\|_{W_{2}^{\lambda}(0, T)}^{2}=\|u\|_{L_{2}(0, T)}^{2}+\int_{0}^{1} \frac{d h}{h^{1+2 \lambda}} \int_{h}^{T}\left|\Delta_{t}(-h) u(t)\right|^{2} d t, \quad 0<\lambda<1,
$$

where $\Delta_{t}(-h) u=u(t-h)-u(t)$, we obtain

$$
\begin{aligned}
& \left\|\theta \boldsymbol{u}_{t}\right\|_{W_{2}^{l, 0}\left(Q_{T}\right)} \leq c \sup _{t<T}\|\theta(\cdot, t)\|_{W_{2}^{l+1}(\Omega)}\left\|\boldsymbol{u}_{t}\right\|_{W_{2}^{l, 0}\left(Q_{T}\right)}, \\
& \left\|\Delta_{t}(-h)\left(\theta \boldsymbol{u}_{t}\right)\right\|_{L_{2}(\Omega)} \\
& \quad \leq c\left(\sup _{\Omega}|\theta(x, t)|\left\|\Delta_{t}(-h) \boldsymbol{u}_{t}\right\|_{L_{2}(\Omega)}+\left\|\boldsymbol{u}_{t}\right\|_{W_{2}^{l}(\Omega)} \int_{0}^{h}\left\|\theta_{t}(\cdot, t-\tau)\right\|_{W_{2}^{1}(\Omega)} d \tau\right),
\end{aligned}
$$

which implies

$$
\left\|\theta \boldsymbol{u}_{t}\right\|_{W_{2}^{l, l / 2}\left(Q_{T}\right)} \leq c\left\|\boldsymbol{u}_{t}\right\|_{W_{2}^{l, l / 2}\left(Q_{T}\right)}\left(\sup _{t<T}\|\theta\|_{W_{2}^{l+1}(\Omega)}+\left\|\theta_{t}\right\|_{W_{2}^{l+1,0}\left(Q_{T}\right)}\right) .
$$

Next, we consider the term $\nabla_{u} \cdot T_{u}-\nabla \cdot T(\boldsymbol{u})=\left(\nabla_{u}-\nabla\right) \cdot T(\boldsymbol{u})+\nabla_{u} \cdot\left(T_{u}(\boldsymbol{u})-T(\boldsymbol{u})\right)$. Since $\nabla_{u}-\nabla=\left(J_{u}^{-1} A-I\right) \nabla$ and

$$
\left\|J_{u}^{-1}-I\right\|_{W_{2}^{l+1}(\Omega)}+\|A-1\|_{W_{2}^{l+1}(\Omega)} \leq c \delta_{1},
$$

we have

$$
\left\|\left(\nabla_{u}-\nabla\right) \cdot T(\boldsymbol{u})\right\|_{W_{2}^{l, 0}\left(Q_{T}\right)} \leq c \delta_{1}\left\|D^{2} \boldsymbol{u}\right\|_{W_{2}^{l, 0}\left(Q_{T}\right)} .
$$

Moreover, from the estimate

$$
\left\|\Delta_{t}(-h)\left(J_{u}^{-1} A-I\right)\right\|_{W_{2}^{1}(\Omega)} \leq c \int_{0}^{h}\|\boldsymbol{u}\|_{W_{2}^{2}(\Omega)} d t \leq c \sqrt{h}\|\boldsymbol{u}\|_{W_{2}^{2,0}\left(Q_{T}\right)},
$$


it follows that

$$
\left\|\left(\nabla_{u}-\nabla\right) \cdot T(\boldsymbol{u})\right\|_{W_{2}^{0, l / 2}\left(Q_{T}\right)} \leq c\left(\delta_{1}+\|\boldsymbol{u}\|_{W_{2}^{2,0}\left(Q_{T}\right)}\right)\left\|D_{x}^{2} \boldsymbol{u}\right\|_{W_{2}^{l, l / 2}\left(Q_{T}\right)} .
$$

The expression $\nabla \cdot\left(T_{u}(\boldsymbol{u})-T(\boldsymbol{u})\right)$ is estimated in a similar way.

Now we estimate the last term $-P(\theta) \nabla \theta+p(\bar{\rho}+\theta)\left(\nabla-\nabla_{u}\right) \theta$ in $\boldsymbol{l}_{1}$, where $P(\theta)=$ $p^{\prime}(\bar{\rho}+\theta)-p^{\prime}(\bar{\rho})$. We have

$$
\|P(\theta) \nabla \theta\|_{L_{2}\left(Q_{T}\right)} \leq c \sup _{Q_{T}}|\theta(x, t)|\|\nabla \theta\|_{L_{2}\left(Q_{T}\right)} .
$$

Assuming that $\theta$ is extended to $\mathbb{R}^{3}$ with preservation of class, we estimate the difference (3.11)

$$
\begin{aligned}
& P(\theta(x+z, t)) \nabla \theta(x+z, t)-P(\theta(x, t)) \nabla \theta(x, t) \\
& \quad=(P(\theta(x+z, t))-P(\theta(x, t)) \nabla \theta(x+z, t)+P(\theta(x, t))(\nabla \theta(x+z, t)-\nabla \theta(x, t)) .
\end{aligned}
$$

Since

$$
\begin{aligned}
|P(\theta(x+z, t))-P(\theta(x, t))| & \leq c|\theta(x+z, t)-\theta(x, t)|, \\
|P(\theta(x, t))| & \leq c|\theta(x, t)|,
\end{aligned}
$$

the $L_{2}$-norm of the difference (3.11) is controlled by

$$
\begin{aligned}
& c\left(\|\theta(x+z, t)-\theta(x, t)\|_{L_{q}(\Omega)}\|\nabla \theta\|_{L_{p}(\Omega)}\right. \\
& \left.\quad+\sup _{\Omega} \mid \theta(x, t)\|\nabla \theta(x+z, t)-\nabla \theta(x, t)\|_{L_{2}(\Omega)}\right) \\
& \leq c\|\theta(x+z, t)-\theta(x, t)\|_{W_{2}^{1}(\Omega)}\|\theta(\cdot, t)\|_{W_{2}^{l+1}(\Omega)} .
\end{aligned}
$$

Multiplying (3.11), (3.12) by $|z|^{-3-2 l}$ and integrating with respect to $z$, we obtain

$$
\|P(\theta) \nabla \theta\|_{W_{2}^{l, 0}\left(Q_{T}\right)} \leq c \sup _{Q_{T}}\|\theta\|_{W_{2}^{l+1}(\Omega)}\|\theta\|_{W_{2}^{l+1,0}\left(Q_{T}\right)} .
$$

The finite difference of $P(\theta) \nabla \theta$ with respect to $t$ is estimated in the same way:

$$
\begin{aligned}
& \| P(\theta) \nabla \theta \|_{W_{2}^{0, l / 2}\left(Q_{T}\right)} \\
& \quad \leq c\left(\sup _{Q_{T}}|\theta(x, t)|\|\nabla \theta\|_{W_{2}^{0, l / 2}\left(Q_{T}\right)}+\|\theta\|_{\left.W_{2}^{l / 2}(0, T), W_{2}^{1}(\Omega)\right)} \sup _{t<T}\|\theta\|_{W_{2}^{l+1}(\Omega)}\right) \\
& \quad \leq c \sup _{t<T}\|\theta\|_{W_{2}^{l+1}(\Omega)}\left(\left\|\theta_{t}\right\|_{W_{2}^{l+1,0}\left(Q_{T}\right)}+\|\theta\|_{W_{2}^{l+1,0}\left(Q_{T}\right)}\right),
\end{aligned}
$$

whence

$$
\|P(\theta) \nabla \theta\|_{W_{2}^{l, l / 2}\left(Q_{T}\right)} \leq c \sup _{t<T}\|\theta\|_{W_{2}^{l+1}(\Omega)}\left(\|\theta\|_{W_{2}^{l+1,0}\left(Q_{T}\right)}+\left\|\theta_{t}\right\|_{W_{2}^{l+1,0}\left(Q_{T}\right)}\right) .
$$

It can be shown by the same arguments that the expression $p(\bar{\rho}+\theta)\left(\nabla_{u}-\nabla\right) \theta$ satisfies the same inequality with the additional term

$$
c \delta_{1}\left(\|\theta\|_{W_{2}^{l+1,0}\left(Q_{T}\right)}+\left\|\theta_{t}\right\|_{W_{2}^{l+1,0}\left(Q_{T}\right)}\right)+c \sup _{t<T}\|\theta\|_{W_{2}^{l+1}(\Omega)}\|\boldsymbol{u}\|_{W_{2}^{2,0}\left(Q_{T}\right)}
$$

on the right hand side.

As for $l_{2}=\theta \nabla_{u} \cdot \boldsymbol{u}+\bar{\rho}\left(\nabla_{u}-\nabla\right) \boldsymbol{u}$, we have

$$
\left\|l_{2}(\boldsymbol{u}, \theta)\right\|_{W_{2}^{l+1,0}\left(Q_{T}\right)} \leq c\left(\delta_{1}+\sup _{t<T}\|\theta\|_{W_{2}^{l+1}(\Omega)}\right)\|\boldsymbol{u}\|_{W_{2}^{l+2,0}\left(Q_{T}\right)} .
$$

Estimate (3.2) is a consequence of (3.6)-(3.16). The proposition is proved.

We describe the procedure of constructing the solution of a nonlinear problem (1.5) in the time interval $t>0$. Observe that the solution of this problem satisfies the condition

$$
\int_{\Omega} \theta(x, t) J_{u}(x, t) d x=0
$$


(in view of the mass conservation), while in the case of a linear problem we have

$$
\int_{\Omega} \theta(x, t) d x=0
$$

Therefore, we shall construct the solution step by step, from the time interval $[(k-$ $1) T, k T]$ to $[k T,(k+1) T]$, with a certain sufficiently large but finite $T$, eliminating at every step the discrepancy between conditions (3.17) and (3.18). This procedure was proposed in 9 .

We set $\boldsymbol{u}=\boldsymbol{u}^{\prime}+\boldsymbol{u}^{\prime \prime}, \theta=\theta^{\prime}+\theta^{\prime \prime}$, represent $\left(\boldsymbol{u}_{0}, \theta_{0}\right)$ in the form $\boldsymbol{u}_{0}=\boldsymbol{u}_{0}^{\prime}+\boldsymbol{u}_{0}^{\prime \prime}$, $\theta_{0}=\theta_{0}^{\prime}+\theta_{0}^{\prime \prime}$, where

$$
\boldsymbol{u}_{0}^{\prime}=\boldsymbol{u}_{0}, \quad \boldsymbol{u}_{0}^{\prime \prime}=0, \quad \theta_{0}^{\prime}=\theta_{0}, \quad \theta_{0}^{\prime \prime}=0,
$$

and define $\left(\boldsymbol{u}^{\prime}, \theta^{\prime}\right)$ and $\left(\boldsymbol{u}^{\prime \prime}, \theta^{\prime \prime}\right)$ as solutions of the problems

$$
\begin{gathered}
\left\{\begin{array}{l}
\bar{\rho} \boldsymbol{u}_{t}^{\prime}-\nabla \cdot T\left(\boldsymbol{u}^{\prime}\right)+p^{\prime}(\bar{\rho}) \nabla \theta^{\prime}=0, \\
\theta_{t}^{\prime}+\bar{\rho} \nabla \cdot \boldsymbol{u}^{\prime}=0, \quad \xi \in \Omega, \\
\boldsymbol{u}^{\prime}(\xi, t)=0, \quad x \in S, \\
\boldsymbol{u}^{\prime}(\xi, 0)=\boldsymbol{u}_{0}(\xi), \quad \theta^{\prime}(\xi, 0)=\theta_{0}(\xi), \quad \xi \in \Omega,
\end{array}\right. \\
\left\{\begin{array}{l}
\bar{\rho} \boldsymbol{u}_{t}^{\prime \prime}-\nabla \cdot T\left(\boldsymbol{u}^{\prime \prime}\right)+p^{\prime}(\bar{\rho}) \nabla \theta^{\prime \prime}=\boldsymbol{l}_{1}\left(\boldsymbol{u}^{\prime}+\boldsymbol{u}^{\prime \prime}, \theta^{\prime}+\theta^{\prime \prime}\right), \\
\theta_{t}^{\prime \prime}+\bar{\rho} \nabla \cdot \boldsymbol{u}^{\prime \prime}=l_{2}\left(\boldsymbol{u}^{\prime}+\boldsymbol{u}^{\prime \prime}, \theta^{\prime}+\theta^{\prime \prime}\right), \quad \xi \in \Omega, \\
\boldsymbol{u}^{\prime \prime}(\xi, t)=0, \quad x \in S, \\
\boldsymbol{u}^{\prime \prime}(\xi, 0)=0, \quad \theta^{\prime \prime}(\xi, 0)=0, \quad \xi \in \Omega,
\end{array}\right.
\end{gathered}
$$

Since $\int_{\Omega} \theta_{0}(x) d x=0$ (because $X(\xi, 0)=I$ and $J_{u}(\xi, 0)=1$ ), problem (3.20) has a global solution satisfying (2.2) (with $\boldsymbol{f}=0, h=0$ ) for arbitrary $T>0$, whereas (3.21) is solvable in the time interval $(0, T)$, when $\epsilon=\epsilon(T)$ in (1.9) is sufficiently small. By $(2.2)$ and (2.55),

$$
\begin{aligned}
e^{\beta T} N\left(\boldsymbol{u}^{\prime}(\cdot, T), \theta^{\prime}(\cdot, T)\right) & \leq c N\left(\boldsymbol{u}_{0}, \theta_{0}\right) \\
Y\left(\boldsymbol{u}^{\prime \prime}, \theta^{\prime \prime}\right) & \left.\leq c\left(\delta_{1} Y\left(\boldsymbol{u}^{\prime \prime}+\boldsymbol{u}^{\prime}, \theta^{\prime \prime}+\theta^{\prime}\right)\right)+Y^{2}\left(\boldsymbol{u}^{\prime \prime}+\boldsymbol{u}^{\prime}, \theta^{\prime \prime}+\theta^{\prime}\right)\right) \\
& \leq c\left(\delta_{1} Y\left(\boldsymbol{u}^{\prime \prime}, \theta^{\prime \prime}\right)+Y^{2}\left(\boldsymbol{u}^{\prime \prime}, \theta^{\prime \prime}\right)+\varepsilon N\left(\boldsymbol{u}_{0}, \theta_{0}\right)\right)
\end{aligned}
$$

(under the assumptions (3.1)), where

$$
N\left(\boldsymbol{u}_{0}, \theta_{0}\right)=\left\|\boldsymbol{u}_{0}\right\|_{W_{2}^{l+1}(\Omega)}+\left\|\theta_{0}\right\|_{W_{2}^{l+1}(\Omega)} .
$$

If $\varepsilon N\left(\boldsymbol{u}_{0}, \theta_{0}\right)$ is sufficiently small, then (3.1) is true, problem (3.21) is solvable for $t \in$ $(0, T)$, and

$$
N\left(\boldsymbol{u}^{\prime \prime}(\cdot, T), \theta^{\prime \prime}(\cdot, T)\right) \leq c Y\left(\boldsymbol{u}^{\prime \prime}, \theta^{\prime \prime}\right) \leq c \varepsilon N\left(\boldsymbol{u}_{0}, \theta_{0}\right)
$$

Hence,

$$
\begin{aligned}
N(\boldsymbol{u}(\cdot, T), \theta(\cdot, T)) & \leq N\left(\boldsymbol{u}^{\prime}(\cdot, T), \theta^{\prime}(\cdot, T)\right)+N\left(\boldsymbol{u}^{\prime \prime}(\cdot, T), \theta^{\prime \prime}(\cdot, T)\right) \\
& \leq c\left(\varepsilon+e^{-\beta T}\right) N\left(\boldsymbol{u}_{0}, \theta_{0}\right) \leq \frac{1}{8} N\left(\boldsymbol{u}_{0}, \theta_{0}\right),
\end{aligned}
$$

provided

$$
c\left(\varepsilon+e^{-\beta T}\right) \leq \frac{1}{8} .
$$

We fix $T$ satisfying this condition.

Assume that the solution of (1.5) is found for $t<k T$, and that

$$
N_{k} \leq \frac{1}{8} N_{k-1} \leq \cdots \leq \frac{1}{8^{k}} N_{0},
$$


where $N_{k}=N(\boldsymbol{u}(\cdot, k T), \theta(\cdot, k T))$. The function $\theta(\xi, k T)$ satisfies

$$
\int_{\Omega} \theta(\xi, k T) J_{u}(\xi, k T) d \xi=0
$$

and we set $\theta(\xi, k T)=\theta_{k}^{\prime}(\xi)+\theta_{k}^{\prime \prime}(\xi)$, where

$$
\begin{aligned}
& \theta_{k}^{\prime}(\xi)=\theta(\xi, k T)-|\Omega|^{-1} \int_{\Omega} \theta(\xi, k T) d \xi, \\
& \theta_{k}^{\prime \prime}(\xi)=|\Omega|^{-1} \int_{\Omega} \theta(\xi, k T)\left(1-J_{u}(\xi, k T)\right) d \xi .
\end{aligned}
$$

We consider problems (3.20) and (3.21) in the interval $t \in(k T,(k+1) T)$, taking $\left(\boldsymbol{u}_{k}^{\prime}=\right.$ $\left.\boldsymbol{u}(\xi, k T), \theta_{k}^{\prime}(\xi)\right)$ and $\left(\boldsymbol{u}_{k}^{\prime \prime}=0, \theta_{k}^{\prime \prime}(\xi)\right)$, respectively, as initial data. It is clear that

$$
\begin{aligned}
N\left(\boldsymbol{u}^{\prime}(\cdot,(k+1) T), \theta^{\prime}(\cdot,(k+1) T)\right. & \leq e^{-\beta T} N\left(\boldsymbol{u}(\cdot, k T), \theta^{\prime}(\cdot, k T)\right) \\
& \leq c e^{-\beta T} N(\boldsymbol{u}(\cdot, k T), \theta(\cdot, k T)),
\end{aligned}
$$

moreover, if conditions (3.1) are satisfied for $t \leq k T$, then we can estimate the nonlinear terms in accordance with $(3.2)$, proving that

$$
N\left(\boldsymbol{u}^{\prime \prime}(\cdot,(k+1) T), \theta^{\prime \prime}(\cdot,(k+1) T)\right) \leq c Y_{k}\left(\boldsymbol{u}^{\prime \prime}, \theta^{\prime \prime}\right) \leq c \delta_{1} N(\boldsymbol{u}(\cdot, k T), \theta(\cdot, k T)),
$$

where $Y_{j}$ is the sum of norms defined in (3.3) and computed in the interval $t \in(j T,(j+$ 1)T). By (3.27) and (3.28),

$$
N(\boldsymbol{u}(\cdot,(k+1) T), \theta(\cdot,(k+1) T)) \leq \frac{1}{8} N(\boldsymbol{u}(\cdot, k T), \theta(\cdot, k T)) .
$$

For every interval $t \in(j T,(j+1) T), j=1, \ldots, k$, we have

$$
Y_{j} \leq\left(Y_{j}^{\prime}+Y_{j}^{\prime \prime}\right) \leq c N_{j} \leq c 8^{-j} N_{0} .
$$

If $\beta$ is so small that $e^{\beta T} \leq 4$, then

$$
\sum_{j=0}^{k} Y_{j}^{2} e^{2 \beta j T} \leq c \sum_{j=0}^{k} \frac{e^{2 \beta j T}}{8^{j}} N_{0}^{2} \leq c N_{0}^{2},
$$

which is equivalent to $(1.10)$.

The verification of condition (3.1) for $\boldsymbol{U}$ in the interval $(0, k T)$ reduces to

$$
\sup _{t<k T}\|\boldsymbol{U}(\cdot, t)\|_{W_{2}^{l+1}(\Omega)} \leq c\left(\int_{0}^{k T} e^{2 \beta t}\|\boldsymbol{u}\|_{W_{2}^{l+2}(\Omega)}^{2} d t\right)^{1 / 2} \leq c N_{0} \leq c \epsilon,
$$

hence we can set $\delta_{1}=c \epsilon$. The inequality $\|\theta\|_{W_{2}^{l+1}(\Omega)} \leq \bar{\rho} / 2$ is verified in a similar way.

More detailed arguments can be found in [10. By the same method, the solution of the nonhomogeneous Navier-Stokes equation with mass forces $\boldsymbol{f}(x, t)$ can be constructed, provided $\boldsymbol{f}$ decays exponentially as $t \rightarrow \infty$ (see [10, 11]).

\section{$\S 4$. ON THE FREE BOUNDARY PROBLEM}

In this section, we consider the free boundary problem

$$
\left\{\begin{array}{l}
\left.\rho(x, t)\left(\boldsymbol{v}_{t}+(\boldsymbol{v} \cdot \nabla) \boldsymbol{v}\right)\right)-\nabla \cdot T(\boldsymbol{v})+\nabla p(\rho)=0, \\
\rho_{t}+\nabla \cdot(\rho \boldsymbol{v})=0, \quad x \in \Omega_{t}^{+} \cup \Omega_{t}^{-}, \quad t>0, \\
{[\boldsymbol{v}]=0, \quad[-p(\rho) \boldsymbol{n}+T(\boldsymbol{v}) \boldsymbol{n}]=0, \quad V_{n}=\boldsymbol{v} \cdot \boldsymbol{n}, \quad x \in \Gamma_{t},} \\
\boldsymbol{v}^{-}(x, t)=0, \quad x \in S, \\
\boldsymbol{v}(x, 0)=\boldsymbol{v}_{0}(x), \quad \rho(x, 0)=\rho_{0}(x), \quad x \in \Omega_{0}^{+} \cup \Omega_{0}^{-},
\end{array}\right.
$$


where $\boldsymbol{v}(x, t)=\boldsymbol{v}^{ \pm}(x, t), \rho(x, t)=\rho^{ \pm}(x, t)$ for $x \in \Omega_{t}^{ \pm}, \Omega_{t}^{+}$and $\Omega_{t}^{-}$are bounded domains separated by a free interface $\Gamma_{t}=\partial \Omega_{t}^{+}$that is given for $t=0$ and should be found for $t>0$. The domain $\Omega=\Omega_{t}^{+} \cup \Gamma_{t} \cup \Omega_{t}^{-}$is fixed; the surface $S=\partial \Omega$ is bounded away from $\Gamma_{t}$. By $T(\boldsymbol{v}) \equiv T^{ \pm}(\boldsymbol{v})$ we mean the viscous part of the stress tensor:

$$
T^{ \pm}(\boldsymbol{v})=\mu^{ \pm} S\left(\boldsymbol{v}^{ \pm}\right)+\mu_{1}^{ \pm} I \nabla \cdot \boldsymbol{v}^{ \pm}, \quad x \in \Omega^{ \pm},
$$

$\boldsymbol{n}$ is the normal to $\Gamma_{t}$ exterior with respect to $\Omega^{+}$, and $V_{n}$ is the velocity of evolution of $\Gamma_{t}$ in the direction $\boldsymbol{n}, \mu^{ \pm}, \mu_{1}^{ \pm}=$const $>0,[\boldsymbol{u}]$ is the jump of $\boldsymbol{u}$ on $\Gamma_{t}$. We assume that $p^{+}(\rho)=p^{-}(\rho)=p(\rho)$.

In the Lagrangian coordinates, problem (4.1) takes the form

$$
\left\{\begin{array}{l}
r(\xi, t) \boldsymbol{u}_{t}-\nabla_{u} \cdot T_{u}(\boldsymbol{u})+\nabla_{u} p(r)=0, \\
r_{t}+r \nabla_{u} \cdot \boldsymbol{u}=0, \quad \xi \in \Omega_{0}^{+} \cup \Omega_{0}^{-}, \quad t>0, \\
{[\boldsymbol{u}]=0, \quad\left[-p(r) \boldsymbol{n}+T_{u}(\boldsymbol{u}) \boldsymbol{n}\right]=0, \quad \xi \in \Gamma_{t},} \\
\boldsymbol{u}^{-}=0, \quad \xi \in S, \\
\boldsymbol{u}(\xi, 0)=\boldsymbol{v}_{0}(\xi), \quad r(\xi, 0)=\rho_{0}(\xi), \quad \xi \in \Omega_{0}^{+} \cup \Omega_{0}^{-},
\end{array}\right.
$$

where $r=\rho(X(\xi, t), t), \boldsymbol{u}(\xi, t)=\boldsymbol{v}(X, t)$,

$$
X(\xi, t)=\xi+\int_{0}^{t} \boldsymbol{u}(\xi, \tau) d \tau \equiv \xi+\boldsymbol{U}(\xi, t), \quad \xi \in \Omega_{0}^{-} \cup \Omega_{0}^{+} .
$$

As in $\S 2$, we set $\nabla_{u}=J_{u}^{-1} A \nabla, S_{u}(\boldsymbol{u})=\left(\nabla_{u} \boldsymbol{u}\right)+\left(\nabla_{u} \boldsymbol{u}\right)^{T}, T_{u}(\boldsymbol{u})=\mu S_{u}(\boldsymbol{u})+\mu_{1} I \nabla_{u} \cdot \boldsymbol{u}$, $J_{u}=\operatorname{det} \mathcal{L}, \mathcal{L}=\left(\frac{\partial X}{\partial \xi}\right), \boldsymbol{n}(X)=\frac{J_{u}^{-1} A \boldsymbol{n}_{0}}{\left|J_{u}^{-1} A \boldsymbol{n}_{0}\right|}$. The elements of the matrix $A$ are co-factors of the elements $a_{i j}=\delta_{i j}+\int_{0}^{t} \frac{\partial u_{i}}{\partial \xi_{j}} d \tau$ of the matrix $\mathcal{L}$.

Moreover, we set $\rho=\bar{\rho}+\theta, \bar{\rho}=M /|\Omega|$,

$$
M=\int_{\Omega} \rho(x, t) d x=\int_{\Omega} r(\xi, t) J_{u}(\xi, t) d \xi=\text { const }>0,
$$

which implies

$$
\int_{\Omega} \theta(\xi, t) J_{u}(\xi, t) d \xi=0
$$

and we write (4.2) in the form

$$
\left\{\begin{array}{l}
\bar{\rho} \boldsymbol{u}_{t}-\nabla \cdot T(\boldsymbol{u})+p^{\prime}(\bar{\rho}) \nabla \theta=\boldsymbol{l}_{1}(\boldsymbol{u}, \theta) \\
\theta_{t}+\bar{\rho} \nabla \cdot \boldsymbol{u}=l_{2}(\boldsymbol{u}, \theta), \quad \xi \in \Omega_{0}^{+} \cup \Omega_{0}^{-} \\
\boldsymbol{u}(\xi, t)=0, \quad \xi \in S, \\
{[\boldsymbol{u}]=0, \quad\left[\mu^{ \pm} \Pi_{0} S(\boldsymbol{u}) \boldsymbol{n}_{0}\right]=\boldsymbol{l}_{3}(\boldsymbol{u}),} \\
{\left[-p^{\prime}(\bar{\rho}) \theta+\boldsymbol{n}_{0} \cdot T(\boldsymbol{u}) \boldsymbol{n}_{0}\right]=l_{4}(\boldsymbol{u}, \theta), \quad \xi \in \Gamma_{0},} \\
\boldsymbol{u}(\xi, 0)=\boldsymbol{v}_{0}(\xi), \quad \theta(\xi, 0)=\theta_{0}(\xi)=\rho_{0}(\xi)-\bar{\rho}, \quad \xi \in \Omega_{0}
\end{array}\right.
$$

where

$$
\begin{aligned}
& \boldsymbol{l}_{1}=-\vartheta \boldsymbol{u}_{t}+\left(\nabla_{u} \cdot T_{u}(\boldsymbol{u})-\nabla \cdot T(\boldsymbol{u})\right)+p^{\prime}(\bar{\rho}) \nabla \theta-p^{\prime}(\bar{\rho}+\theta) \nabla_{u} \theta, \\
& l_{2}=-r \nabla_{u} \cdot \boldsymbol{u}+\bar{\rho} \nabla \cdot \boldsymbol{u}, \\
& \boldsymbol{l}_{3}=\left[\mu\left(\Pi_{0}^{2} S(\boldsymbol{u}) \boldsymbol{n}_{0}-\Pi_{0} \Pi S_{u}(\boldsymbol{u}) \boldsymbol{n}\right)\right], \\
& l_{4}=-\left[\boldsymbol{n} \cdot T_{u}(\boldsymbol{u}) \boldsymbol{n}-\boldsymbol{n}_{0} \cdot T(\boldsymbol{u}) \boldsymbol{n}_{0}\right]+\left[p(\bar{\rho}+\theta)-p(\bar{\rho})-p^{\prime}(\bar{\rho}) \theta\right], \\
& \quad \Pi \boldsymbol{f}=\boldsymbol{f}-\boldsymbol{n}(\boldsymbol{f} \cdot \boldsymbol{n}), \quad \Pi_{0} \boldsymbol{f}=\boldsymbol{f}-\boldsymbol{n}_{0}\left(\boldsymbol{f} \cdot \boldsymbol{n}_{0}\right) .
\end{aligned}
$$


Along with (4.3), we consider the linear problem

$$
\left\{\begin{array}{l}
\bar{\rho} \boldsymbol{v}_{t}-\nabla \cdot T(\boldsymbol{v})+p^{\prime}(\bar{\rho}) \nabla \theta=\boldsymbol{f}(x, t), \\
\theta_{t}+\bar{\rho} \nabla \cdot \boldsymbol{v}=h(x, t), \quad \xi \in \Omega_{0}^{+} \cup \Omega_{0}^{-}, \quad t>0, \\
\boldsymbol{v}(x, t)=0, \quad x \in S, \\
{[\boldsymbol{v}]=0, \quad\left[-p^{\prime}(\bar{\rho}) \theta \boldsymbol{n}_{0}+T(\boldsymbol{u}) \boldsymbol{n}_{0}\right]=\boldsymbol{b}(x, t), \quad x \in \Gamma_{0},} \\
\boldsymbol{v}(x, 0)=\boldsymbol{v}_{0}(x), \quad \theta(x, 0)=\theta_{0}(x), \quad x \in \Omega .
\end{array}\right.
$$

The main attention is paid to the analysis of problem (4.5). We state analogs of Theorems 2, 3 and Proposition 1.

Theorem 4. 1. For arbitrary $\boldsymbol{v}_{0} \in W_{2}^{l+1}\left(\Omega^{ \pm}\right), \theta_{0} \in W_{2}^{l+1}\left(\Omega^{ \pm}\right), \boldsymbol{f} \in W_{2}^{l, l / 2}\left(Q_{T}^{ \pm}\right)$, $h \in W_{2}^{l+1,0}\left(Q_{T}^{ \pm}\right)$, and $\boldsymbol{b} \in W_{2}^{l+1 / 2, l / 2+1 / 4}\left(G_{T}\right)$ satisfying the conditions

$$
\begin{aligned}
& \boldsymbol{v}_{0}(x)=0, \quad x \in S, \\
& {\left[\boldsymbol{v}_{0}\right]=0, \quad\left[-p^{\prime}(\bar{\rho}) \theta_{0} \boldsymbol{n}_{0}+T\left(\boldsymbol{u}_{0}\right) \boldsymbol{n}_{0}\right]=\boldsymbol{b}(x, 0), \quad x \in \Gamma_{0},}
\end{aligned}
$$

problem (4.5) has a unique solution $\boldsymbol{v} \in W_{2}^{2+l, 1+l / 2}\left(Q_{T}^{ \pm}\right), \theta, \theta_{t} \in W_{2}^{l+1,0}\left(Q_{T}^{ \pm}\right), T<\infty$, and

$$
\begin{aligned}
\sum_{ \pm}\left(\|\boldsymbol{v}\|_{W_{2}^{l+2, l / 2+1}\left(Q_{T}^{ \pm}\right)}+\|\theta\|_{W_{2}^{l+1,0}\left(Q_{T}^{ \pm}\right)}+\left\|\theta_{t}\right\|_{W_{2}^{l+1,0}\left(Q_{T}^{ \pm}\right)}\right) & \\
\leq c(T)\left(\sum _ { \pm } \left(\left\|\boldsymbol{v}_{0}\right\|_{W_{2}^{l+1}\left(\Omega^{ \pm}\right)}\right.\right. & +\left\|\theta_{0}\right\|_{W_{2}^{l+1}\left(\Omega^{ \pm}\right)}+\|\boldsymbol{f}\|_{W_{2}^{l, l / 2}\left(Q_{T}^{ \pm}\right)} \\
& \left.\left.+\|h\|_{W_{2}^{l+1,0}\left(Q_{T}^{ \pm}\right)}\right)+\|\boldsymbol{b}\|_{W_{2}^{l+1 / 2, l / 2+1 / 4}\left(G_{T}\right)}\right)
\end{aligned}
$$

where $G_{T}=\Gamma_{0} \times(0, T)$.

2. Moreover, if

$$
\int_{\omega} \theta_{0}(x) d x=0, \quad \int_{\Omega} h(x, t) d x=0, \quad t>0,
$$

then the solution is defined for all $t>0$, the condition $\int_{\Omega} \theta(x, t) d x=0$ is fulfilled, and

$$
\begin{aligned}
\sum_{ \pm}\left(\left\|e^{\beta t} \boldsymbol{v}\right\|_{W_{2}^{l+2, l / 2+1}\left(Q_{T}^{ \pm}\right)}+\left\|e^{\beta t} \theta\right\|_{W_{2}^{l+1,0}\left(Q_{T}^{ \pm}\right)}+\left\|e^{\beta t} \theta_{t}\right\|_{W_{2}^{l+1,0}\left(Q_{T}^{ \pm}\right)}\right) \\
\leq c\left(\sum _ { \pm } \left(\left\|\boldsymbol{v}_{0}\right\|_{W_{2}^{l+1}\left(\Omega^{ \pm}\right)}+\left\|\theta_{0}\right\|_{W_{2}^{l+1}\left(\Omega^{ \pm}\right)}+\left\|e^{\beta t} \boldsymbol{f}\right\|_{W_{2}^{l, l / 2}\left(Q_{T}^{ \pm}\right)}\right.\right. \\
\left.\left.+\left\|e^{\beta t} h\right\|_{W_{2}^{l+1,0}\left(Q_{T}^{ \pm}\right)}\right)+\left\|e^{\beta t} \boldsymbol{b}\right\|_{W_{2}^{l+1 / 2, l / 2+1 / 4}\left(G_{T}\right)}\right)
\end{aligned}
$$

with $\beta>0$ and $c$ independent of $T \leq \infty$.

Proposition 3. If (4.8) is true, then the solution of problem (4.5) satisfies the condition $\int_{\Omega} \theta(x, t) d x=0, t \geq 0$, and

$$
\begin{aligned}
& \sup _{t<T} e^{2 \beta t}\left(\|\boldsymbol{v}(\cdot, t)\|_{L_{2}(\Omega)}^{2}+\mid \theta(\cdot, t) \|_{L_{2}(\Omega)}^{2}\right)+\int_{0}^{T} e^{2 \beta t}\left(\|\boldsymbol{v}\|_{W_{2}^{1}(\Omega)}^{2}+\|\theta\|_{L_{2}(\Omega)}^{2}\right) d t \\
& \quad \leq c\left(\left\|\boldsymbol{v}_{0}\right\|_{L_{2}(\Omega)}^{2}+\left\|\theta_{0}\right\|_{L_{2}(\Omega)}^{2}+\int_{0}^{T} e^{2 \beta t}\left(\|\boldsymbol{f}\|_{L_{2}(\Omega)}^{2}+\|h\|_{L_{2}(\Omega)}^{2}+\|\boldsymbol{b}\|_{L_{2}\left(\Gamma_{0}\right)}^{2}\right) d t\right) .
\end{aligned}
$$

Estimate (4.10) is proved in the same way as (2.2). Now we briefly describe the proof of (4.9) by the method of localization.

Let $\left\{\omega_{k}\right\}$ be a covering of $\Omega$ of the following form: the $\omega_{k}, k=1, \ldots, m^{-}-1$ are the balls $\left|x-x_{k}\right| \leq d$ with $x_{k} \in S$ (as in $\S 2$ ), the $\omega_{k}, k=1+m^{-}, \ldots, m^{-}+m^{+}-1$, are the balls $\left|x-x_{k}\right| \leq d$ with $x_{k} \in \Gamma_{0}$; the $\omega_{m^{-}}$and $\omega_{m^{-}+m^{+}}$are strictly interior 
subdomains of $\Omega^{-}$and $\Omega^{+}$, respectively. Let $\left\{\varphi_{k}\right\}$ be a partition of unity subordinate to this covering, and let $\zeta_{k}, k=1, \ldots, m^{-}-1, k=m^{-}+1, \ldots, m^{-}+m^{+}-1$, be functions with $\operatorname{supp} \zeta_{k} \subset B_{k} \subset \omega_{k} \cap \omega_{m^{-}}$or $B_{k} \subset \omega_{k} \cap \omega_{m^{-}+m^{+}}$such that $\int_{B_{k}} \zeta_{k}(x) d x=1$, where the $B_{k}$ are balls with radius $\alpha d, \alpha<1$. We set $\boldsymbol{v}_{k}=\boldsymbol{v} \zeta_{k}, \theta_{k}=\theta \zeta_{k}, \omega_{k}^{\prime}=\omega_{k} \cap \Omega$, and

$$
\boldsymbol{w}_{k}(x, t)=\boldsymbol{v}_{k}(x, t)-\zeta_{k}(x) \int_{\omega_{k}^{\prime}} \boldsymbol{v}_{k}(y, t) d y, \quad \vartheta_{k}=\theta_{k}-\zeta_{k} \int_{\omega_{k}^{\prime}} \theta_{k}(y, t) d y
$$

for $k \neq m^{-}, m^{-}+m^{+}$, and

$$
\begin{aligned}
& \widetilde{\boldsymbol{w}}_{m^{-}}(x, t)=\boldsymbol{v}_{m^{-}}(x, t)+\sum_{k=1}^{m^{-}-1} \zeta_{k}(x) \int_{\omega_{k}^{\prime}} \boldsymbol{v}_{k}(y, t) d y, \\
& \widetilde{\boldsymbol{w}}_{m^{-}+m^{+}}=\boldsymbol{v}_{m^{-}+m^{+}}+\sum_{k=m^{-}+1}^{m^{-}+m^{+}-1} \zeta_{k} \int_{\omega_{k}^{\prime}} \boldsymbol{v}_{k} d y, \\
& \widetilde{\vartheta}_{m^{-}}(x, t)=\theta_{m^{-}}(x, t)+\sum_{k=1}^{m^{-}-1} \zeta_{k}(x) \int_{\omega_{k}^{\prime}} \theta_{k}(y, t) d y, \\
& \widetilde{\vartheta}_{m^{-}+m^{+}}=\theta_{m^{-}+m^{+}}+\sum_{k=m^{-}+1}^{m^{-}+m^{+}-1} \zeta_{k} \int_{\omega_{k}^{\prime}} \theta_{k}(y) d y .
\end{aligned}
$$

Moreover, let $\widetilde{\omega}=\Omega \backslash\left(\bar{\omega}_{m^{-}} \cup \bar{\omega}_{m^{-}+m^{+}}\right), \widetilde{\omega}_{m^{-}}=\omega_{m^{-}} \cup \widetilde{\omega}, \widetilde{\omega}_{m^{-}+m^{+}}=\omega_{m^{-} m^{+}} \cup \widetilde{\omega}$, and let

$$
\begin{aligned}
\boldsymbol{w}_{m^{-}}(x, t) & =\widetilde{\boldsymbol{w}}_{m^{-}}-\widetilde{\zeta}(x) \int_{\widetilde{\omega}_{m^{-}}} \widetilde{\boldsymbol{w}}_{m^{-}}(y, t) d y, \\
\boldsymbol{w}_{m^{-}+m^{+}}(x, t) & =\widetilde{\boldsymbol{w}}_{m^{-}+m^{+}}+\widetilde{\zeta}(x) \int_{\widetilde{\omega}_{m^{-}}} \widetilde{\boldsymbol{w}}_{m^{-}}(y, t) d y, \\
\vartheta_{m^{-}}(x, t) & =\widetilde{\vartheta}_{m^{-}}-\widetilde{\zeta}(x) \int_{\widetilde{\omega}_{m^{-}}} \widetilde{\vartheta}_{m^{-}}(y, t) d y, \\
\vartheta_{m^{-}+m^{+}}(x, t) & =\widetilde{\vartheta}_{m^{-}+m^{+}}+\widetilde{\zeta}(x) \int_{\widetilde{\omega}_{m^{-}}} \widetilde{\vartheta}_{m^{-}}(y, t) d y,
\end{aligned}
$$

where $\widetilde{\zeta}(x)$ is a smooth function with $\operatorname{supp} \widetilde{\zeta} \subset \widetilde{\omega}$ and $\int_{\widetilde{\omega}} \widetilde{\zeta}(x) d x=1$. It is clear that

$$
\begin{aligned}
& \int_{\omega_{k}^{\prime}} \vartheta_{k}(x, t) d x=0, \quad k \neq m^{-}, m^{-}+m^{+}, \\
& \int_{\omega_{m^{-}}} \tilde{\vartheta}_{m^{-}} d x+\int_{\omega_{m^{-}+m^{+}}} \widetilde{\vartheta}_{m^{-}+m^{+}} d x=0, \\
& \int_{\widetilde{\omega}_{m^{-}}} \vartheta_{m^{-}} d x=0, \quad \int_{\widetilde{\omega}_{m^{-}+m^{+}}} \vartheta_{m^{-}+m^{+}} d x=0 .
\end{aligned}
$$

As in $\S 2$, we have

$$
\begin{gathered}
\bar{\rho} \boldsymbol{w}_{k, t}-\nabla \cdot T\left(\boldsymbol{w}_{k}\right)+p^{\prime}(\bar{\rho}) \nabla \vartheta_{k}=\boldsymbol{f}_{k}+\mathfrak{f}_{k}, \\
\vartheta_{k, t}+\bar{\rho} \nabla \cdot \boldsymbol{w}_{k}=h_{k}+\mathfrak{h}_{k}, \quad k \neq m^{-}, m^{-}+m^{+}, \\
\bar{\rho} \boldsymbol{w}_{m^{-}, t}-\nabla \cdot T\left(\boldsymbol{w}_{m^{-}}\right)+p^{\prime}(\bar{\rho}) \nabla \vartheta_{m^{-}}=\boldsymbol{f}_{m^{-}}+\mathfrak{f}_{m^{-}}, \\
\vartheta_{m^{-}, t}+\bar{\rho} \nabla \cdot \boldsymbol{w}_{m^{-}}=h_{m^{-}}+\mathfrak{h}_{m^{-}}, \\
\bar{\rho} \boldsymbol{w}_{m^{-}+m^{+}, t}-\nabla \cdot T\left(\boldsymbol{w}_{m^{-}+m^{+}}\right)+p^{\prime}(\bar{\rho}) \nabla \vartheta_{m^{-}+m^{+}}=\boldsymbol{f}_{m^{-}+m^{+}}+\mathfrak{f}_{m^{-}+m^{+}}, \\
\vartheta_{m^{-}+m^{+}, t}+\bar{\rho} \nabla \cdot \boldsymbol{w}_{m^{-}+m^{+}}=h_{m^{-}+m^{+}}+\mathfrak{h}_{m^{-}+m^{+}},
\end{gathered}
$$


where

$$
\begin{aligned}
& \boldsymbol{f}_{k}=\boldsymbol{f} \varphi_{k}-\zeta_{k} \int_{\omega_{k}^{\prime}} \boldsymbol{f} \varphi_{k} d x, \quad h_{k}=h \varphi_{k}-\zeta_{k} \int_{\omega_{k}^{\prime}} h \varphi_{k} d x, \quad k \neq m^{-}, m^{-}+m^{+}, \\
& \tilde{\boldsymbol{f}}_{m^{-}}=\boldsymbol{f} \varphi_{m^{-}}+\sum_{k=1}^{m^{-}-1} \zeta_{k} \int_{\omega_{k}^{\prime}} \boldsymbol{f} \varphi_{k} d x, \quad \tilde{h}_{m^{-}}=h \varphi_{m^{-}}+\sum_{k=1}^{m^{-}-1} \zeta_{k} \int_{\omega_{k}^{\prime}} h \varphi_{k} d x \\
& \tilde{\boldsymbol{f}}_{m^{-}+m^{+}}=\boldsymbol{f} \varphi_{m^{-}+m^{+}}+\sum_{k=1+m^{-}}^{m^{-}+m^{+}-1} \zeta_{k} \int_{\omega_{k}^{\prime}} \boldsymbol{f} \varphi_{k} d x \\
& \widetilde{h}_{m^{-}+m^{+}}=h \varphi_{m^{-}+m^{+}}+\sum_{k=1+m^{-}}^{m^{-}+m^{+}-1} \zeta_{k} \int_{\omega_{k}^{\prime}} h \varphi_{k} d x \\
& \boldsymbol{f}_{m^{-}}(x, t)=\tilde{\boldsymbol{f}}_{m^{-}}(x, t)-\widetilde{\zeta}(x) \int_{\widetilde{\omega}_{m^{-}}} \tilde{\boldsymbol{f}}_{m^{-}} d y \\
& \boldsymbol{f}_{m^{-}+m^{+}}(x, t)=\tilde{\boldsymbol{f}}_{m^{-}+m^{+}}(x, t)+\widetilde{\zeta}(x) \int_{\widetilde{\omega}_{m^{-}}} \tilde{\boldsymbol{f}}_{m^{-}} d y, \\
& h_{m^{-}}=\widetilde{h}_{m^{-}}-\tilde{\zeta}(x) \int_{\widetilde{\omega}_{m^{-}}} \widetilde{h}_{m^{-}} d y, \quad h_{m^{-}+m^{+}}=\widetilde{h}_{m^{-}+m^{+}}+\widetilde{\zeta}(x) \int_{\widetilde{\omega}_{m^{-}}} \tilde{h}_{m^{-}} d y .
\end{aligned}
$$

The functions $\mathfrak{f}_{k}, \mathfrak{h}_{k}$ involve derivatives of the cut-off functions $\varphi_{k}, \zeta_{k}$ and, consequently, only lower order derivatives of $\boldsymbol{v}$ and $\theta$.

In order to estimate $\boldsymbol{v}, \theta$ in the interior of $\Omega^{ \pm}$or in the neighborhood of $S$, we need to analyze equations (4.12) and (4.13) completed by the initial conditions for $\boldsymbol{w}_{k}, \vartheta_{k}$, or equations (4.11) completed by the initial and boundary conditions $\boldsymbol{w}_{k}=0, x \in S$. This is done as in $\S 2$.

To estimate $\boldsymbol{v}, \theta$ near $\Gamma_{0}$, we turn to equations (4.11) with $k=m^{-}+1, \ldots, m^{-}+m^{+}-1$, completed by the conditions

$$
\begin{aligned}
& {\left[\boldsymbol{w}_{k}\right]=0, \quad\left[-p^{\prime}(\bar{\rho}) \vartheta_{k} \boldsymbol{n}_{0}+T\left(\boldsymbol{w}_{k}\right) \boldsymbol{n}_{0}\right]=\boldsymbol{b}_{k}+\mathfrak{b}_{k}, \quad x \in \Gamma_{0}} \\
& \boldsymbol{w}_{k}(x, 0)=\boldsymbol{v}_{k}(x, 0)-\zeta_{k}(x) \int_{\omega_{k}^{\prime}} \boldsymbol{v}_{k}(\eta, 0) d \eta \equiv \boldsymbol{w}_{0}^{(k)}(x), \\
& \vartheta_{k}(x, 0)=\theta_{k}(x, 0)-\zeta_{k}(x) \int_{\omega_{k}^{\prime}} \theta_{k}(\eta, 0) d \eta \equiv \vartheta_{0}^{(k)}(x)
\end{aligned}
$$

with $\boldsymbol{b}_{k}(x, t)=\boldsymbol{b}(x, t) \varphi_{k}(x)$ and $\mathfrak{b}_{k}$ containing lower order derivatives of $\boldsymbol{v}$ and $\theta$. After "rectifying" $\Gamma_{0}$ near $x_{k}$ and "freezing" the coefficients at this point, we arrive at the following model problem (an analog of (2.28)):

$$
\left\{\begin{array}{l}
\bar{\rho} \boldsymbol{w}_{t}-\nabla \cdot T(\boldsymbol{w})+p^{\prime}(\bar{\rho}) \nabla \vartheta=\boldsymbol{f}+\tilde{\mathfrak{f}}, \\
\vartheta_{t}+\bar{\rho} \nabla \cdot \boldsymbol{w}=h+\widetilde{\mathfrak{h}}, \quad y \in \widehat{\mathfrak{Q}}, \\
{[\boldsymbol{w}]=0, \quad\left[\mu S_{\alpha 3}(\boldsymbol{w})\right]=b_{\alpha}+\widetilde{\mathfrak{b}}_{\alpha}, \quad \alpha=1,2,} \\
{\left[-p^{\prime}(\bar{\rho}) \vartheta+T_{33}(\boldsymbol{w})\right]=b_{3}+\widetilde{\mathfrak{b}}_{3}, \quad y_{3}=0, \quad y^{\prime} \in \mathfrak{Q}^{\prime},} \\
\boldsymbol{w}(y, t)=0, \quad y_{3}= \pm 2 d_{0}, \quad y^{\prime} \in \mathfrak{Q}^{\prime}, \\
\boldsymbol{w}(y, 0)=\boldsymbol{w}_{0}(y), \quad \vartheta(y, 0)=\vartheta_{0}(y), \quad y \in \widehat{\mathfrak{Q}},
\end{array}\right.
$$

where

$$
\widehat{\mathfrak{Q}}=\mathfrak{Q}^{\prime} \times\left(-2 d_{0}, 2 d_{0}\right), \quad \mathfrak{Q}^{\prime}=\left\{\left|y_{\alpha}\right| \leq d_{0}, y_{3}=0\right\}, \quad \alpha=1,2 .
$$


We take the finite differences of (4.16) with respect to $y_{1}, y_{2}$ and, like in $\S 2$, obtain the following estimate similar to (2.34):

$$
\begin{aligned}
& \left\|e^{\beta t} \nabla \boldsymbol{w}\right\|_{W_{2, \tan }^{l+1,0}\left(\hat{\mathfrak{Q}}_{T}\right)}^{2}+\left\|e^{\beta t} \boldsymbol{w}\right\|_{W_{2, \tan }^{l+1,0}\left(\hat{\mathfrak{Q}}_{T}\right)}^{2}+\left\|e^{\beta t} \vartheta\right\|_{W_{2, \tan }^{l+1}\left(\hat{\mathfrak{Q}}_{T}\right)}^{2} \\
& \leq c\left(\left\|e^{\beta t}(\boldsymbol{f}+\tilde{\mathfrak{f}})\right\|_{W_{2, \tan }^{l, 0}\left(\hat{\mathfrak{Q}}_{T}\right)}^{2}+\left\|e^{\beta t}(h+\widetilde{\mathfrak{h}})\right\|_{W_{2, \tan }^{l+1,0}\left(\hat{\mathfrak{Q}}_{T}\right)}^{2}\right. \\
& \quad+\left\|e^{\beta t}(\boldsymbol{b}+\widetilde{\mathfrak{b}})\right\|_{W_{2}^{l+1 / 2,0}\left(\mathfrak{Q}_{T}^{\prime}\right)}^{2}+\|\boldsymbol{w}(\cdot, 0)\|_{W_{2, \tan }^{l+1}(\hat{\mathfrak{Q}})}^{2}+\|\vartheta(\cdot, 0)\|_{\left.W_{2, \tan (\hat{\mathfrak{Q}})}^{l+1}\right)}^{2} .
\end{aligned}
$$

To estimate the derivatives of $\boldsymbol{w}$ and $\vartheta$ with respect to $t$ and $y_{3}$, we treat (4.16) as a union of one-dimensional problems (cf. (2.35), (2.36)):

$$
\begin{gathered}
\left\{\begin{array}{l}
\bar{\rho} w_{\alpha, t}-\mu \frac{\partial^{2} w_{\alpha}}{\partial y_{3}^{2}}=g_{\alpha}, \quad\left|y_{3}\right|<2 d_{0}, \quad \alpha=1,2 \\
{\left[w_{\alpha}\right]=0, \quad\left[\mu \frac{\partial w_{\alpha}}{\partial y_{3}}\right]=a_{\alpha}\left(y^{\prime}, t\right), \quad y_{3}=0} \\
w_{\alpha}(y, t)=0, \quad y_{3}= \pm 2 d_{0}, \\
w_{\alpha}(y, 0)=w_{\alpha 0}(y), \quad\left|y_{3}\right| \leq 2 d_{0},
\end{array}\right. \\
\left\{\begin{array}{l}
\bar{\rho} w_{3, t}-\left(2 \mu+\mu_{1}\right) \frac{\partial^{2} w_{3}}{\partial y_{3}^{2}}+p^{\prime}(\bar{\rho}) \frac{\partial \vartheta}{\partial y_{3}}=g_{3}, \\
\vartheta_{t}+\bar{\rho} \frac{\partial w_{3}}{\partial y_{3}}=e, \quad\left|y_{3}\right|<2 d_{0}, \\
{\left[w_{3}\right]=0, \quad\left[-p^{\prime}(\bar{\rho}) \vartheta+\left(2 \mu+\mu_{1}\right) \frac{\partial w_{3}}{\partial y_{3}}\right]=a_{3}, \quad y_{3}=0} \\
w_{3}=0, \quad y_{3}= \pm 2 d_{0}, \\
w_{3}(y, 0)=w_{30}(y), \quad \vartheta(y, 0)=\vartheta_{0}(y), \quad\left|y_{3}\right| \leq 2 d_{0} .
\end{array}\right.
\end{gathered}
$$

Both $w_{\alpha}$ and $\left(w_{3}, \vartheta\right)$ can be constructed and estimated as in $\S 2$. Consider problem (4.19). We introduce the function $u_{1}(x, t)=u_{1}^{ \pm}(x, t), x \in I^{ \pm}=\left\{ \pm x_{3} \in\left(0,2 d_{0}\right)\right\}$ satisfying the conditions

$$
u_{1}^{ \pm}(x, 0)=w_{3,0}^{ \pm}(x), \quad x_{3} \in I^{ \pm}, \quad u_{1}^{ \pm}(x, t)=0, \quad x_{3}= \pm 2 d_{0}
$$

$u_{1}^{ \pm}(x, t)=0$ for $t>2$, and the inequality

$$
\left\|u_{1}^{ \pm}\right\|_{W_{2}^{l+2, l / 2+1}\left(I^{ \pm} \times(0,2)\right)} \leq c\left\|w_{3,0}\right\|_{W_{2}^{l+1}\left(I^{ \pm}\right)} .
$$

It follows that

$$
\sum_{ \pm}\left\|u_{1}^{ \pm}\right\|_{L_{2}\left(\mathfrak{Q}^{\prime}, W_{2}^{2+l, 1+l / 2}\left(I^{ \pm} \times(0,2)\right)\right)} \leq\left\|w_{3}\right\|_{L_{2}\left(\mathfrak{Q}^{\prime}, W_{2}^{1+l}\left(I^{ \pm}\right)\right)} .
$$

The function $\vartheta^{(1)}(x, t)=\vartheta_{0}(x) \chi(t)$ satisfies

$$
\sum_{ \pm}\left(\left\|\vartheta^{(1)}\right\|_{W_{2}^{l+1,0}\left(I^{ \pm} \times(0,2)\right)}+\left\|\vartheta_{t}^{(1)}\right\|_{W_{2}^{l+1,0}\left(I^{ \pm} \times(0,2)\right)}\right) \leq c \sum_{ \pm}\left\|\vartheta_{0}\right\|_{W_{2}^{l+1}\left(I^{ \pm}\right)} .
$$

For the differences $u_{2}=w_{3}-u_{1}, \vartheta^{2)}=\vartheta-\vartheta^{(1)}$ we obtain the problem

$$
\left\{\begin{array}{l}
\bar{\rho} u_{2, t}-\left(2 \mu+\mu_{1}\right) \frac{\partial^{2} u_{2}}{\partial y_{3}^{2}}+p^{\prime}(\bar{\rho}) \frac{\partial \vartheta^{(2)}}{\partial y_{3}}=g_{3}^{(1)} \\
\vartheta_{t}^{(2)}+\bar{\rho} \frac{\partial u_{2}}{\partial y_{3}}=e^{(1)}, \quad\left|y_{3}\right|<2 d_{0} \\
{\left[w_{3}\right]=0, \quad\left[-p^{\prime}(\bar{\rho}) \vartheta^{(2)}+\left(2 \mu+\mu_{1}\right) \frac{\partial u_{2}}{\partial y_{3}}\right]=a_{3}^{(1)}, \quad y_{3}=0} \\
w_{3}=0, \quad y_{3}= \pm 2 d_{0} \\
u_{2}(y, 0)=0, \quad \vartheta^{(2)}(y, 0)=0, \quad\left|y_{3}\right| \leq 2 d_{0}
\end{array}\right.
$$


with $g_{3}^{(1)}, e^{(1)}, a^{(1)}$ satisfying

$$
\begin{gathered}
\sum_{ \pm}\left(\left\|e^{\beta t} g^{(1)}\right\|_{W_{2}^{l, l / 2}\left(I^{ \pm} \times(0, T)\right)}+\left\|e^{\beta t} e^{(1)}\right\|_{W_{2}^{l+1,0}\left(I^{ \pm} \times(0, T)\right)}\right)+\left\|e^{\beta t} a^{(1)}\right\|_{W_{2}^{l / 2+1 / 4}(0, T)} \\
\leq c\left(\sum_{ \pm}\left(\left\|e^{\beta t} g\right\|_{W_{2}^{l, l / 2}\left(I^{ \pm} \times(0, T)\right)}+\left\|e^{\beta t} e\right\|_{W_{2}^{l+1,0}\left(I^{ \pm} \times(0, T)\right)}\right)+\left\|e^{\beta t} a\right\|_{W_{2}^{l / 2+1 / 4}(0, T)}\right. \\
\quad+\sum_{ \pm}\left(\left\|e^{\beta t} u_{1}\right\|_{W_{2}^{2+l, 1+l / 2}\left(I^{ \pm} \times(0, T)\right)}+\left\|e^{\beta t} \vartheta^{(1)}\right\|_{W_{2}^{l+1,0}\left(I^{ \pm} \times(0, T)\right)}\right) \\
\left.+\left\|e^{\beta t} \vartheta_{t}^{(1)}\right\|_{W_{2}^{l+1,0}\left(I^{ \pm} \times(0, T)\right)}\right) .
\end{gathered}
$$

By applying the Laplace transformation and eliminating $\vartheta^{(2)}$, we convert (4.20) into the Sturm-Liouville type problem similar to (2.41):

$$
\left\{\begin{array}{l}
R(s) \widetilde{u}_{2}-\frac{d^{2} \widetilde{u}_{2}}{d y_{3}^{2}}=\widetilde{G}, \quad y_{3} \in I^{ \pm} \\
{\left[\widetilde{u}_{2}\right]=0, \quad\left[\lambda(s) \frac{d \widetilde{u}_{2}}{d y_{3}}\right]=\widetilde{A}} \\
u_{2}=0, \quad y_{3}= \pm 2 d_{0}
\end{array}\right.
$$

where $\lambda^{ \pm}(s)=\frac{a^{ \pm} s+b}{a_{0} s+b}, a_{0}>0$,

$$
R(s)=R^{ \pm}(s)=\frac{\bar{\rho} s^{2}}{a^{ \pm} s+b}, \quad a^{ \pm}=2 \mu^{ \pm}+\mu_{1}^{ \pm}, \quad b=\bar{\rho} p^{\prime}(\bar{\rho}),
$$

and $G$ and $A$ are functions satisfying

$$
\begin{aligned}
& \sum_{ \pm}\left\|e^{\beta t} G\right\|_{W_{2}^{l, l / 2}\left(I^{ \pm} \times(0, T)\right)}^{2}+\left\|e^{\beta t} A\right\|_{W_{2}^{1 / 2+l / 2}(0, T)} \\
& \leq c\left(\sum_{ \pm}\left(\left\|e^{\beta t} g_{3}^{(1)}\right\|_{W_{2}^{l, l / 2}\left(I^{ \pm} \times(0, T)\right)}^{2}+\left\|e^{\beta t} e^{(1)}\right\|_{W_{2}^{l+1,0}\left(I^{ \pm} \times(0, T)\right)}\right)+\left\|e^{\beta t} a_{3}^{(1)}\right\|_{W_{2}^{l / 2+1 / 4}(0, T)}\right) .
\end{aligned}
$$

The solution of (4.21) can be expressed as the sum $\widetilde{u}_{2}=\widetilde{u}_{3}+\widetilde{u}_{4}$, with $\widetilde{u}_{3}, \widetilde{u}_{4}$ defined by

$$
R(s) \widetilde{u}_{3}-\frac{d^{2} \widetilde{u}_{3}}{d y_{3}^{2}}=\widetilde{G}, \quad y_{3} \in I^{ \pm}, \quad \widetilde{u}_{3}=0, \quad y_{3}=0, \pm 2 d_{0}
$$

and

$$
\left\{\begin{array}{l}
R(s) \widetilde{u}_{4}-\frac{d^{2} \widetilde{u}_{4}}{d y_{3}^{2}}=0, \quad y_{3} \in I^{ \pm} \\
\widetilde{u}_{4}=0, \quad y_{3}=0, \pm 2 d_{0}, \\
{\left[\widetilde{u}_{4}\right]=0, \quad\left[\lambda(s) \frac{d \widetilde{u}_{4}}{d y_{3}}\right]=\widetilde{A}^{(1)}=\widetilde{A}-\left[\lambda(s) \frac{d \widetilde{u}_{3}}{d y_{3}}\right], \quad y_{3}=0}
\end{array}\right.
$$

The functions $\widetilde{u}_{3}^{ \pm}$are given by a formula similar to (2.43), and

$$
\begin{aligned}
\widetilde{u}_{4}\left(y_{3}\right) & =C_{1}\left(e^{r^{+}\left(2 d_{0}-y_{3}\right)}-e^{-r^{+}\left(2 d_{0}-y_{3}\right)}\right), \quad y_{3} \in I^{+}, \\
\widetilde{u}_{4}\left(y_{3}\right) & =C_{2}\left(e^{-r^{-}\left(2 d_{0}+y_{3}\right)}-e^{r^{-}\left(2 d_{0}+y_{3}\right)}\right), \quad y_{3} \in I^{-}, \\
C_{1} & =-D^{-1}\left(e^{2 d_{0} r^{-}}-e^{-2 d_{0} r^{-}}\right) \widetilde{A}^{(1)}, \\
C_{2} & =-D^{-1}\left(e^{2 d_{0} r^{+}}-e^{-2 d_{0} r^{+}}\right) \widetilde{A}^{(1)},
\end{aligned}
$$

where $r^{ \pm}(s)= \pm \sqrt{R(s)}$ and

$D=\lambda^{-} r^{-}\left(e^{-2 d_{0} r^{-}}+e^{2 d_{0} r^{-}}\right)\left(e^{2 d_{0} r^{+}}-e^{-2 d_{0} r^{+}}\right)+\lambda^{+} r^{+}\left(e^{2 d_{0} r^{-}}-e^{-2 d_{0} r^{-}}\right)\left(e^{2 d_{0} r^{+}}+e^{-2 d_{0} r^{+}}\right)$. 
It can be shown that

$$
\begin{gathered}
\sum_{ \pm}\left\|e^{\beta t} u_{3}\right\|_{W_{2}^{2+l, 1+l / 2}\left(I^{ \pm} \times(0, T)\right)}^{2} \leq c \sum_{ \pm}\left\|e^{\beta t} G\right\|_{W_{2}^{l, l / 2}\left(I^{ \pm} \times(0, T)\right)}^{2}, \\
\sum_{ \pm}\left\|e^{\beta t} u_{4}\right\|_{W_{2}^{2+l, 1+l / 2}\left(I^{ \pm} \times(0, T)\right)}^{2} \leq c\left(\left\|e^{\beta t} A\right\|_{W_{2}^{1 / 4+l / 2}(0, T)}^{2}+\sum_{ \pm}\left\|e^{\beta t} u_{3}\right\|_{W_{2}^{2+l, 1+l / 2}\left(I^{ \pm} \times(0, T)\right)}^{2}\right)
\end{gathered}
$$

The functions $w_{\alpha}$ are estimated in a similar way, so finally we arrive at the inequality

$$
\begin{aligned}
\sum_{ \pm}\left(\left\|e^{\beta t} \boldsymbol{w}_{k}\right\|_{W_{2}^{2+l, 1+l / 2}\left(\mathfrak{Q}_{T}^{ \pm}\right)}^{2}+\left\|e^{\beta t} \vartheta_{k}\right\|_{W_{2}^{1+l, 0}\left(\mathfrak{Q}_{T}^{ \pm}\right)}^{2}+\left\|e^{\beta t} \vartheta_{k, t}\right\|_{W_{2}^{1+l, 0}\left(\mathfrak{Q}_{T}^{ \pm}\right)}^{2}\right) \\
\leq \sum_{ \pm}\left(\left\|e^{\beta t} \boldsymbol{g}_{k}\right\|_{W_{2}^{l, l / 2}\left(\mathfrak{Q}_{T}^{ \pm}\right)}^{2}+\left\|e^{\beta t} e_{k}\right\|_{W_{2}^{1+l, 0}\left(\mathfrak{Q}_{T}^{ \pm}\right)}^{2}+\left\|e^{\beta t} \boldsymbol{a}_{k}\right\|_{W_{2}^{l+1 / 2, l / 2+1 / 4}\left(\mathfrak{Q}_{T}^{\prime \pm}\right)}^{2}\right. \\
\left.+\left\|\boldsymbol{w}_{k}(\cdot, 0)\right\|_{W_{2}^{l+1}\left(\mathfrak{Q}^{ \pm}\right)}^{2}+\left\|\vartheta_{k}(\cdot, 0)\right\|_{W_{2}^{l+1}\left(\mathfrak{Q}^{ \pm}\right)}^{2}\right)
\end{aligned}
$$

for the solution $\left(\boldsymbol{w}_{k}, \vartheta_{k}\right)$ of $(4.18),(4.19)$. Taking account of the interior estimates of $\left(\boldsymbol{w}_{k}, \vartheta_{k}\right)$ and estimates near $S$, we complete the proof of (4.9).

We restrict ourselves to the above comments concerning Theorem 4 . Because of the lack of space, we omit the arguments related to the estimates of nonlinear terms (4.4) and construction of the solution of problem (4.3). They are similar to the corresponding arguments in $\S 3$. As a result, we prove the following theorem.

Theorem 5. Suppose $S, \Gamma_{0} \in W_{2}^{3 / 2+l}, 1 / 2<l<1, p \in C^{2}(\bar{\rho} / 2,3 \bar{\rho} / 2), \bar{\rho}, p^{\prime}(\bar{\rho})>0$. For arbitrary $\boldsymbol{v}_{0} \in W_{2}^{l+1}\left(\Omega_{0}^{ \pm}\right)$and $\rho_{0} \in W_{2}^{l+1}\left(\Omega_{0}^{ \pm}\right)$satisfying the conditions

$$
\begin{aligned}
& \boldsymbol{v}_{0}(x, 0)=0, \quad x \in S, \quad \int_{\Omega_{0}} \theta_{0}(x) d x=0, \\
& {\left[\boldsymbol{v}_{0}\right]=0, \quad\left[-p\left(\rho_{0}\right) \boldsymbol{n}_{0}+T\left(\boldsymbol{u}_{0}\right) \boldsymbol{n}_{0}\right]=0, \quad x \in \Gamma_{0},} \\
& \left|\rho_{0}\right| \geq c_{0}>0,
\end{aligned}
$$

$$
\sum_{ \pm}\left\|\boldsymbol{v}_{0}\right\|_{W_{2}^{l+1}\left(\Omega^{ \pm}\right)}+\sum_{ \pm}\left\|\theta_{0}^{ \pm}\right\|_{W_{2}^{l+1}\left(\Omega^{ \pm}\right)} \leq \epsilon \ll 1
$$

problem (4.3) has a unique solution defined for $t \geq 0$, and

$$
\begin{aligned}
\sum_{ \pm}\left(\left\|e^{\beta t} \boldsymbol{v}\right\|_{W_{2}^{l+2, l / 2+1}\left(Q_{\infty}^{ \pm}\right)}+\left\|e^{\beta t} \theta^{ \pm}\right\|_{W_{2}^{l+1,0}\left(Q_{\infty}^{ \pm}\right)}+\left\|e^{\beta t} \theta_{t}^{ \pm}\right\|_{W_{2}^{l+1,0}\left(Q_{\infty}^{ \pm}\right)}\right) \\
\leq c \sum_{ \pm}\left(\left\|\boldsymbol{v}_{0}\right\|_{W_{2}^{l+1}\left(\Omega^{ \pm}\right)}+\left\|\theta_{0}\right\|_{W_{2}^{l+1}\left(\Omega^{ \pm}\right)}\right) .
\end{aligned}
$$

\section{REFERENCES}

[1] M. Padula, On the exponential stability of the rest state of a viscous compressible fluid, J. Math. Fluid Mech. 1 (1999), no. 1, 62-77. MR 1699019

[2] _ Asymptotic stability of steady compressible fluids, Lecture Notes in Math., vol. 2024, Springer, Heidelberg, 2011, pp. 1-229. MR2848254

[3] I. V. Denisova, On energy inequality for the problem on the evolution of two fluids of different types without surface tension, J. Math. Fluid Mech. 17 (2015), no. 1, 183-198. MR3313115

[4] O. A. Ladyzhenskaya and V. A. Solonnikov, Some problems of vector analysis and generalized formulations of boundary value problems for the Navier-Stokes equation, Zap. Nauchn. Sem. Leningrad. Otdel. Mat. Inst. Steklov. (LOMI) 59 (1976), 81-116. (Russian) MR0467031

[5] M. E. Bogovskiǔ, Solutions of some problems of vector analysis, associated with the operators Div and Grad, Tr. Sem. S. L. Soboleva 1 (1980), 5-40. (Russian) MR631691

[6] L. P. Galdi, An introduction to the mathematical theory of the Navier-Stokes equations. Vol. 1, Linear steady problems, Springer Tracts Nat. Philos., vol. 38, Springer-Verlag, New York, 1994. MR 1284205 
[7] K. K. Golovkin, On equivalent normalizations of fractional spaces, Tr. Mat. Inst. Steklova 66 (1962), 364-383. (Russian) MR.0154028

[8] V. I. Smirnov, A course of higher mathematics. Vol. IV, Integral equations and partial differential equations, GITTL, Moscow, 1953; English transl., Pergamon Press, Oxford-New York, 1964. MR 0177069

[9] V. A. Solonnikov, On problem of stability of equilibrium figures of uniformly rotating viscous incompressible liquid, Instability in Models Connected with Fluid Flows. II, Int. Math. Ser. (N.Y.), vol. 7, Springer, New York, 2008, 189-254. MR2459267

[10] E. V. Frolova, Free boundary problem of magnetohydrodynamics, Zap. Nauchn. Sem. S.-Peterburg. Otdel. Mat. Inst. Steklov. (POMI) 425 (2014), 149-178; English transl., J. Math. Sci. (N.Y.) 210 (2015), no. 6, 857-877. MR.2749373

[11] I. V. Denisova, Global $L_{2}$-solvability of a problem governing two-phase fluid motion without surface tension, Portugal. Math. 71 (2014), no. 1, 1-24. MR.3194642

[12] V. A. Solonnikov and A. Tani, Free boundary problem for a viscous compressible flow with a surface tension, Constantin Caratheodory: an international tribute. Vol. II, World Sci. Publ. Co., 1991, pp. 1270-1303. MR 1130887

[13] A. Matsumura and T. Nishida, The initial-boundary value problem for the equations of motion of compressible viscous and heat conducting fluids, Proc. Japan Acad., Ser. A 55 (1979), no. 9, 337-342. MR.555060

[14] _ Initial-boundary value problems for the equations of motion of compressible viscous and heat conducting fluids, Comm. Math. Physics 89 (1983), no. 4, 445-464. MR713680

[15] G. Ströhmer, About the resolvent of an operator from fluid dynamics, Math. Z. 194 (1987), no. 2, 183-191. MR876229

[16] _ About compressible fluid in a bounded region, Pacific J. Math. 143 (1990), no. 2, 359-375. MR 1051082

[17] W. M. Zajaczkowski, On non-stationary motion of a compressible barotropic viscous fluid bounded by a free surface, Diss. Math. (Rozprawy Mat.) 324 (1993), 1-101. MR1218047

[18] _ On non-stationary motion of a compressible barotropic viscous fluid bounded by a free surface, SIAM J. Math. Anal. 25 (1994), no. 1, 1-84. MR.1257142

[19] Y. Enomoto and Y. Shibata, On the $\mathcal{R}$-sectoriality and the initial boundary value problem for the viscous compressible fluid flow, Funkcialaj Ekvac. 56 (2013), no. 3, 441-505. MR3157151

[20] M. S. Agranovich and M. I. Vishik, Elliptic problems with a parameter and parabolic problems of general type, Uspekhi Mat. Nauk 19 (1964), no. 3, 53-161; English transl., Russian Math. Surveys 19 (1964), no. 3, 53-157. MR0192188

St. Petersburg Branch, Steklov Mathematical Institute, Russian Academy of Sciences, Fontanka 27, 191023, St. Petersburg, Russia

E-mail address: solonnik@pdmi.ras.ru

Received 2/DEC/2014

Originally published in English 